Biometrika (2019), xx, x, pp. 1-31

Printed in Great Britain

\title{
A kernel regression model for panel count data with time-varying coefficients
}

\author{
BY YANG WANG \\ Department of Statistics, School of Mathematical Sciences and SJTU-Yale Joint Center for \\ Biostatistics, Shanghai Jiao Tong University, Shanghai, China. \\ wy910028@sjtu.edu.cn \\ ZHANGSHENG YU PH.D \\ Department of Statistics, School of Mathematical Sciences and SJTU-Yale Joint Center for \\ Biostatistics, Shanghai Jiao Tong University, Shanghai, China. \\ yuzhangsheng@sjtu.edu.cn
}

\begin{abstract}
SUMMARY
For the conditional mean function of panel count model with time-varying coefficients, we propose to use local kernel regression method for estimation. Partial log-likelihood with local polynomial is formed for estimation. Under some regularity conditions, strong uniform consistency rates are obtained for the local estimator. At target time point, we show that the local estimator converges in distribution to normal distribution. The baseline mean function estimator is also shown to be consistent. Simulation studies show that the time-varying coefficient estimator is close to the true value, the empirical coverage probabilities of the confidence interval is close to the nominal level. We also applied the proposed method to analyze a clinical study on childhood wheezing.
\end{abstract}

Some key words: kernel weight, local partial log-likelihood, local kernel estimating equation.

\section{INTRODUCTION}

Panel count data occurred when events are observed at finite fixed time points and the visit times vary from subject to subject, and the exact event times between two consecutive observation times are unknown. In reality, panel count data are often encountered in clinical, demographical and industrial researches. For example, in an observational study on childhood asthma (Tepper et al. (2008)), the number of wheezing episodes experienced by each child between two consecutive interviews were collected by phone call. The event number may be greater than one, and the exact time of wheezing occurrence was unknown. The wheezing event time analysis should be considered as the panel count data type. Meanwhile, the risk factors' effect on the panel count outcome may vary over time. Therefore, it is desirable to study the panel count model with nonparametric time-varying coefficients.

In the past three decades, there have been extensive researches to study the proportional mean model for panel count data. Generally, there are two main approaches, one is pseudo-likelihood estimation method, and the other is the estimating equation approach. For the likelihood method, pseudo-likelihood function was constructed based on the nonhomogeneous Poisson process assumptions, see Zhang (2002), Wellner \& Zhang (2007). Zhu et al. (2018) developed a likelihood-based semi-parametric regression model for panel count data under the same assumptions. Lei et al. (2014) proposed sieve maximum likelihood method under the Gamma-Frailty inhomogeneous Poisson process assumption. For the estimating equation approach, Hu et al. (2003), Sun et al. (2007) and Li et al. (2010) discussed estimating equation approach to analyze the semi-parametric regression model for panel count data with correlated observa- 
tion times. He et al. (2007), Li et al. (2011) and Li et al. (2015) proposed estimating equation approach for regression analysis of multivariate panel count data. All above methods focused on the parametric covariate effect estimation which lead to bias estimators when the covariate effect changes over time. Therefore, statistical methods dealing with time-varying coefficient for panel count data are much desired.

In this paper, we focus on the nonparametric time-varying coefficient estimation for panel count data. For nonparametric regression model, there are two main approaches, kernel estimation and spline method, generally used to study for survival data in recent researches. For example, Cai \& Sun (2003), Tian et al. (2005), Cai et al. (2007), Yu \& Lin (2010) and Lin et al. (2016) discussed kernel-weighted likelihood method for Cox model with time-varying effects. Buchholz \& Sauerbrei (2011), Perperoglou (2013) and Perperoglou (2014) proposed B-spline methods for time-varying effects model in survival data analysis. Nevertheless, limited work has been done in nonparametric panel count model. Zhao et al. (2018) investigated B-splined pseudo-likelihood method for time-varying coefficients model of panel count data. However, they only provided the asymptotic distribution for an integral of the estimator instead of the proposed estimator. Furthermore, the robustness of splined estimation was poor depending on the knot numbers. To make up for these deficiencies, we employ the kernel estimated approach to study timevarying coefficients panel count model. Under the assumption of nonhomogeneous Poisson process, we construct kernel-weighted local partial log-likelihood for estimation and provide the asymptotic properties of the coefficient estimators.

In the following, $\S 2$ presents time-varying coefficients mean model, and the kernel-weighted local partial log-likelihood for estimation. $\S 3$ derives the asymptotic theoretical properties of estimators based on modern empirical process theories. $\S 4$ describes the numerical results obtained from simulation studies to exam the proposed model. $\S 5$ applies the proposed approach to a child wheeze study. $\S 6$ concludes the paper with a discussion. The technical details are presented in Appendices.

\section{THE MEAN FUNCTION MODEL AND LOCAL PARTIAL LOG-LIKELIHOOD ESTIMATION}

\subsection{The conditional mean function model}

We first introduce some notations. Let $\left\{N_{i}(t), t \geq 0\right\}$ be a counting process of the cumulative number of events up to time $t, 0 \leq t \leq \tau$, and $\tau$ is the maximum follow up time. Without loss of generality, we assume that $N_{i}(0)=0, i=1,2, \ldots, n$. For subject $i$, the patient is followed at time $\left\{T_{i l}: 0<T_{i 1}<T_{i 2}<\right.$ $\left.\cdots<T_{i k_{i}}<\infty\right\}$, where $k_{i}$ and $T_{i l}$ are random. We denote $\left\{O_{i}(t), t \geq 0\right\}$ as the observation process, which is a point process $O_{i}(t)=\sum_{l=1}^{k_{i}} I\left(T_{i l} \leq t\right), t \geq 0$, representing the cumulative visit numbers up to time $t$. Here, $I(\cdot)$ is the indicator function. Let $o_{i}(t)=O_{i}(t)-O_{i}(t-)$, so that $o_{i}(t)$ denotes whether subject $i$ has a visit at time $t$. Suppose that $C_{i}, i=1,2, \ldots, n$, are censoring times. And $N_{i}\left(T_{i l}\right)$ is not observed when $C_{i}<T_{i l}<\tau$. Also let $\left\{Z_{i}, i=1, \ldots, n\right\}$ be $d$-dimensional covariates. In this paper, for simplicity, we consider $d=1$. Suppose that given $Z_{i}$, the mean function of $N_{i}(t)$ is

$$
E\left\{N_{i}(t) \mid Z_{i}=z_{i}\right\}=\mu_{0}(t) \exp \left(\beta(t) z_{i}\right), \quad t \geq 0,
$$

where the baseline function $\mu_{0}(t)$ is unspecified, and $\beta(t)$ is an unknown function. In this study, we assume that $\left\{N_{i}(t), O_{i}(t), C_{i}, Z_{i}\right\}, i=1, \ldots, n$, are independent and identically distribution. Furthermore, we assume that $N_{i}(t), O_{i}(t)$ and $C_{i}$ are independent, given the covariate $Z_{i}$.

\subsection{Local kernel estimation conditional on observation process}

As the information about recurrent process $N_{i}(t)$ can be observed at visit times, we define a new counting process $\tilde{N}_{i}(t)$, with respect to subject $i$, conditional on observation process:

$$
\widetilde{N}_{i}(t)=\int_{0}^{t} N_{i}(u) d O_{i}(u), \quad t \geq 0
$$

The defined process only jumps at the observation times $\left\{T_{i, l}, l=1, \ldots, k_{i}\right\}$, and the jump size is $N_{i}\left(T_{i, l}\right)$. Then, conditional on the observation process $O_{i}(t)$ and covariate $Z_{i}$, the mean of $d \widetilde{N}_{i}(t)$ is 
as follows:

$$
E\left\{d \widetilde{N}_{i}(t) \mid Z_{i}=z_{i} ; O_{i}(u), 0<u \leq t\right\}=\mu_{0}(t) \exp \left(\beta(t) z_{i}\right) d O_{i}(t) .
$$

Suppose that $d \widetilde{N}_{i}(t)$ is nonhomogeneous Poisson process, we can construct the logarithm of partial likelihood function with observed information over $[0, \tau](\tau>0)$ by employing similar techniques presented in work of Lawless \& Nadeau (1995) and Hu et al. (2003), as follows:

$$
p l_{n}(\beta(u))=n^{-1} \sum_{i=1}^{n} \int_{0}^{\tau} I\left(C_{i} \geq u\right)\left\{\beta(u) z_{i}-\log n^{-1} \sum_{j=1}^{n} I\left(C_{j} \geq u\right) \exp \left(\beta(u) z_{j}\right) o_{j}(u)\right\} d \widetilde{N}_{i}(u) .
$$

To estimate the time-varying coefficient, we employ the kernel likelihood approach. For each fixed time point $t$, using Taylor expansion, we approximate the $\beta(u)$ with the $p$ th-order polynomial as:

$$
\beta(u) \approx \beta(t)+\beta^{\prime}(t)(u-t)+\cdots+\beta^{(p)}(t)(u-t)^{p} / p !,
$$

Set $\boldsymbol{\beta}=\left(\beta_{0}(t), \beta_{1}(t), \ldots, \beta_{p}(t)\right)^{\mathrm{T}}=\left(\beta(t), \beta^{\prime}(t), \ldots, \beta^{(p)}(t) / p !\right)^{\mathrm{T}}$ and $z_{i}(\boldsymbol{u})=z_{i}(1, u-t, \ldots,(u-$ $\left.t)^{p}\right)^{\mathrm{T}}$. Let $K(\cdot)$ be a kernel function which can down weight the likelihood contribution of remote time points, and let $h$ be the bandwidth that can regulate the local neighborhood sizes. Then, by inserting localizing weights, with the local polynomial equation (5), we obtain the local partial log-likelihood:

$$
\begin{aligned}
& \mathcal{L}_{n}(\boldsymbol{\beta})= \\
& n^{-1} \sum_{i=1}^{n} \int_{0}^{\tau} K_{h}(u-t) I\left(C_{i} \geq u\right)\left\{\boldsymbol{\beta}^{\mathrm{T}} z_{i}(\boldsymbol{u})-\log n^{-1} \sum_{j=1}^{n} I\left(C_{j} \geq u\right) \exp \left(\boldsymbol{\beta}^{\mathrm{T}} z_{j}(\boldsymbol{u})\right) o_{j}(u)\right\} d \widetilde{N}_{i}(u),
\end{aligned}
$$

where $K_{h}(\cdot)=h^{-1} K(\cdot / h)$.

Let $\widehat{\boldsymbol{\beta}}$ be the maximizer of (6) with respect to $\boldsymbol{\beta}$. Then $\widehat{\beta}(t)=\widehat{\beta}_{0}(t)$ is the local kernel partial maximum likelihood estimator of $\beta(t)$, which is the first component of vector $\widehat{\boldsymbol{\beta}}$.

To obtain the maximizer of (6), we introduce some additional notations. Let

$$
\widetilde{S}_{n, j}(u, \boldsymbol{\beta})=n^{-1} \sum_{i=1}^{n} I\left(C_{i} \geq u\right) \exp \left(\boldsymbol{\beta}^{\mathrm{T}} z_{i}(\boldsymbol{u})\right) o_{i}(u) z_{i}(\boldsymbol{u})^{\otimes j}, \quad j=0,1,2 .
$$

Then, (6) can be modified as follows:

$$
\mathcal{L}_{n}(\boldsymbol{\beta})=n^{-1} \sum_{i=1}^{n} \int_{0}^{\tau} K_{h}(u-t) I\left(C_{i} \geq u\right)\left\{\boldsymbol{\beta}^{\mathrm{T}} z_{i}(\boldsymbol{u})-\log \widetilde{S}_{n, 0}(u, \boldsymbol{\beta})\right\} d \widetilde{N}_{i}(u) .
$$

And we can derive the local kernel estimating equation,

$$
\mathcal{L}_{n}^{\prime}(\boldsymbol{\beta})=n^{-1} \sum_{i=1}^{n} \int_{0}^{\tau} K_{h}(u-t) I\left(C_{i} \geq u\right)\left\{z_{i}(\boldsymbol{u})-\widetilde{S}_{n, 1}(u, \boldsymbol{\beta}) / \widetilde{S}_{n, 0}(u, \boldsymbol{\beta})\right\} d \widetilde{N}_{i}(u) .
$$

which is the gradient of $\mathcal{L}_{n}(\boldsymbol{\beta})$. Again, the Hessian matrix of $\mathcal{L}_{n}(\boldsymbol{\beta})$ is formed as

$$
\begin{aligned}
& \mathcal{L}_{n}^{\prime \prime}(\boldsymbol{\beta})= \\
& -n^{-1} \sum_{i=1}^{n} \int_{0}^{\tau} K_{h}(u-t) I\left(C_{i} \geq u\right)\left\{\widetilde{S}_{n, 2}(u, \boldsymbol{\beta}) / \widetilde{S}_{n, 0}(u, \boldsymbol{\beta})-\left(\widetilde{S}_{n, 1}(u, \boldsymbol{\beta}) / \widetilde{S}_{n, 0}(u, \boldsymbol{\beta})\right)^{\otimes 2}\right\} d \widetilde{N}_{i}(u) .
\end{aligned}
$$

By Cauchy-Schwarz inequality, we can check that the right-hand side of (10) is negative, as $n \rightarrow \infty$. Thus, $\mathcal{L}_{n}(\boldsymbol{\beta})$ is strictly concave with respect to $\boldsymbol{\beta}$. Hence, there is a unique maximizer of the local likelihood 
$\mathcal{L}_{n}(\boldsymbol{\beta})$. Then, using the Newton-Raphson algorithm, we can get the local kernel estimator $\widehat{\boldsymbol{\beta}}$. Here, the $(j+1)$ th step of Newton-Raphson algorithm is

$$
\widehat{\boldsymbol{\beta}}^{(j+1)}=\widehat{\boldsymbol{\beta}}^{(j)}-\mathcal{L}_{n}^{\prime}\left(\widehat{\boldsymbol{\beta}}^{(j)}\right) / \mathcal{L}_{n}^{\prime \prime}\left(\widehat{\boldsymbol{\beta}}^{(j)}\right),
$$

where $\widehat{\boldsymbol{\beta}}^{(j)}$ is the value at $j$ th iteration.

After obtaining the $\widehat{\beta}(t)=\widehat{\beta}_{0}(t)$ at each observation time, we can construct the Breslow type estimator $\widehat{\mu}_{0}(t)$ for the baseline mean function $\mu_{0}(t)$ as $\widehat{\mu}_{0}(t)=\sum_{i=1}^{n} I\left(C_{i} \geq t\right) N_{i}(t) o_{i}(t) / \sum_{i=1}^{n} I\left(C_{i} \geq\right.$ $t) \exp \left(\beta(t) z_{i}\right) o_{i}(t)$ (Breslow (1974) and Cox (1992)). Substituting $\beta(t)$ by $\widehat{\beta}(t)$, we obtain the baseline estimator

$$
\widehat{\mu}_{0}(t, \widehat{\beta}(t))=\sum_{i=1}^{n} I\left(C_{i} \geq t\right) N_{i}(t) o_{i}(t) / \sum_{i=1}^{n} I\left(C_{i} \geq t\right) \exp \left(\widehat{\beta}(t) z_{i}\right) o_{i}(t)
$$

\section{ASYMPTOTIC PROPERTIES}

\subsection{Strong uniform consistency and asymptotic normality}

In this section, we present the asymptotic theoretical properties of the proposed estimator. For simplicity of presentation, we introduce some notations. Let $\boldsymbol{u}=\left(1, u, \ldots, u^{p}\right)^{\mathrm{T}}, \Omega_{1}=\int K(u) \boldsymbol{u}^{\otimes 2} d u$, $\Omega_{2}=\int K^{2}(u) \boldsymbol{u}^{\otimes 2} d u$. Set $H=\operatorname{diag}\left(1, h, \ldots, h^{p}\right), \boldsymbol{u}-\boldsymbol{t}=\left(1,(u-t) / h, \ldots,(u-t)^{p} / h^{p}\right)^{\mathrm{T}}$, and the true value $\boldsymbol{\beta}^{*}=\left(\beta(t), \beta^{\prime}(t) \ldots, \beta^{(p)}(t) / p !\right)^{\mathrm{T}}$.

$p_{1}(t \mid z)=\operatorname{pr}(C \geq t \mid Z=z), \quad p_{2}(t \mid z)=\operatorname{pr}(o(t) \mid Z=z), \quad \mu(t \mid z)=\mu_{0}(t) \exp (\beta(t) z)$, Define$$
\sigma(t \mid z)=\mu_{0}^{2}(t) \exp (2 \beta(t) z), \quad q_{j}(t)=E\left(p_{1}(t \mid z) p_{2}(t \mid z) \mu(t \mid z) z^{j}\right), \quad j=0,1,2 .
$$

$$
\sigma_{1}(t)=q_{2}(t)-q_{1}^{2}(t) / q_{0}(t), \quad \sigma_{2}(t)=E\left(p_{1}(t \mid z) p_{2}(t \mid z)\left(z-q_{1}(t) / q_{0}(t)\right)^{2} \sigma(t \mid z)\right) .
$$

Let $T=\{t: t \in[0, \tau]\}$.

The following regularity conditions are required for the theorems and lemmas.

Condition 1 . The kernel function $K(\cdot) \geq 0$ is a symmetric density function with compact support $[-1,1]$, and is bounded variation taking the value as zero at the boundaries;

Condition 2. $N(\cdot), O(\cdot)$ are bounded, $E\left(N^{2}(\cdot) \mid Z=z\right)$ is exist and $E\left(Z^{\lambda}\right)^{1 / \lambda}<\infty$, for $2<\lambda<\infty$;

Condition 3. The time-varying coefficient $\beta(t)$ is $(p+1)$ th-order continuous differentiable with bounded variation in $T$;

Condition 4. $\mu_{0}(t), p_{1}(t \mid z), p_{2}(t \mid z)$ and $\beta(t) z$ are positive and continuous in $T$;

Condition 5. $q_{0}(t)>0, q_{1}(t), q_{2}(t), \sigma_{1}(t)$ and $\sigma_{2}(t)$ are continuous, and $\inf \sigma_{1}(t)=M_{1}<\infty$, $\sup q_{1}(t) / q_{0}(t)=M_{2}<1, \sup q_{0}(t)=M_{3}<\infty$.

The above conditions will be used to prove the strong uniform consistency and pointwise asymptotic normality of the proposed estimator. Conditions 1-3 are technical and regularity conditions. Conditions 4 and 5 are necessary for deriving the uniform convergence result. Among them, we assume $p_{1}(t \mid z)>0$ and $p_{2}(t \mid z)>0$, which ensure that there is at least one event on each $t \in T$ as $n$ gets large enough. This is crucial to theoretical demonstration of asymptotic properties. Next up, we state the main results of this paper. The detailed proofs are relegated to Appendices.

THEOREM 1. Under Conditions 1-5, assume that the bandwidth h satisfies the conditions:

$$
h \rightarrow 0, \quad n h / \log n \rightarrow \infty \quad \text { and } \quad h \geq(\log n / n)^{1-2 / \lambda} \quad \text { for } \quad \lambda>2,
$$


then there exists a sequence of solutions $\left\{\widehat{\boldsymbol{\beta}}=\left(\widehat{\beta}_{0}(t), \ldots, \widehat{\beta}_{p}(t)\right)^{\mathrm{T}}\right\}$ to equation (9), such that, for each $k=0, \ldots, p$, almost surely

$$
\sup _{t \in T}\left|\widehat{\beta}_{k}(t)-\beta^{(k)}(t) / k !\right|=O\left(h^{-k}\left\{(\log n /(n h))^{1 / 2}+h\right\}\right) \quad \text { as } \quad n \rightarrow \infty .
$$

Especially, when the local linear approximation is used $(p=1)$, we have, almost surely

$$
\sup _{t \in T}|\widehat{\beta}(t)-\beta(t)|=O\left((\log n /(n h))^{1 / 2}+h\right) \quad \text { as } n \rightarrow \infty .
$$

The above theorem shows that the proposed estimator is strong uniformly consistent. This indicates the local estimator is uniform asymptotically unbiased as $n \rightarrow \infty$. Under more stringent conditions, the strong uniform consistency rate of the proposed estimator is similar to that of Zhao (1994) and Claeskens \& Van Keilegom (2003). In their paper, they discussed the strong uniform convergence rate for the nonparametric location regression problem. Here, the strong uniform consistency of the proposed estimator is derived based on Lemma B1 and Lemma B2 presented in Appendix 2. In particular, Lemma B1 discusses the supremum of the local kernel estimating equation (9) under some conditions, which play a crucial role in the proof of Theorem 1. The detailed proofs are presented in Appendix 2.

THEOREM 2. Under Conditions 1-5, assume that the bandwidth h satisfies the conditions:

$$
h \rightarrow 0, \quad n h \rightarrow \infty, \quad \text { and } n h^{2 p+3} \text { is bounded, }
$$

then the asymptotic distribution of $\widehat{\boldsymbol{\beta}}$ satisfies

$$
(n h)^{1 / 2}\left\{H\left(\widehat{\boldsymbol{\beta}}-\boldsymbol{\beta}^{*}\right)-\Omega_{1}^{-1} \boldsymbol{b} h^{p+1} \beta^{(p+1)}(t) /(p+1) !\right\} \rightarrow N\left(0, \sigma_{1}^{-2}(t) \sigma_{2}(t) \Omega_{1}^{-1} \Omega_{2} \Omega_{1}^{-1}\right),
$$

where $\boldsymbol{b}=\int u^{p+1} \boldsymbol{u} K(u) d u$.

The result in above theorem demonstrates the asymptotic normality of the proposed estimator, under general conditions. The $\widehat{\boldsymbol{\beta}}$ converges in the optimal rate of kernel estimators and analogous to the spline estimator. The bias is of order $h^{p+1}$ and related to the $(p+1)$-derivative of real function $\beta(t)$. Hence, it tends to zero when the bandwidth gets to zero. The theorem also gives the joint asymptotic normality of the estimator for derivatives. Particularly, the variance and bias of $\widehat{\beta}^{(r)}(t)=\widehat{\beta}_{r}(t)$ can be obtained by the $r$ th component of (15). The detailed proof is presented in Appendix 2. We also present the two lemmas which are key to the proof of Theorem 1 and Theorem 2 in Appendix 1. When the local linear approximation is used $(p=1)$, we have the following corollary:

COROLLARY 1. Under Conditions 1-5, and assume that the bandwidth h satisfies the conditions:

$$
h \rightarrow 0, \quad n h \rightarrow \infty, \text { and } n h^{5} \text { is bounded, }
$$

then the asymptotic distribution of $\widehat{\beta}(t)$ satisfies

$$
(n h)^{1 / 2}\left\{\widehat{\beta}(t)-\beta(t)-2^{-1} \mu_{2} h^{2} \beta^{\prime \prime}(t)\right\} \rightarrow N\left(0, \nu_{0} \sigma_{1}^{-2}(t) \sigma_{2}(t)\right),
$$

where $\mu_{2}=\int u^{2} K(u) d u, \nu_{0}=\int K^{2}(u) d u$.

The estimator of nonparametric $\beta(t)$ is asymptotically normal. The bias is of order $h^{2}$ and related to the second derivative of time-varying function $\beta(t)$. As consequence of (16), by minimizing the weighted mean integrated squared error:

$$
\int_{0}^{\tau}\left\{4^{-1} \mu_{2}^{2} h^{4} \beta^{\prime \prime 2}(t)+\nu_{0} \sigma_{1}^{-2}(t) \sigma_{2}(t) /(n h)\right\} w(t) d t,
$$

we can derive the theoretical optimal bandwidth for $\widehat{\beta}(t)$, as follows:

$$
h_{\text {opt }}=\left\{\nu_{0} \int_{0}^{\tau} \sigma_{1}^{-2}(t) \sigma_{2}(t) w(t) d t /\left(\mu_{2}^{2} \int_{0}^{\tau} \beta^{\prime \prime 2}(t) w(t) d t\right)\right\}^{1 / 5} n^{-1 / 5} .
$$




\subsection{Estimation of covariance matrix}

We propose the covariance estimator of $\widehat{\boldsymbol{\beta}}$ based on its asymptotic covariance by plugging the estimated $\boldsymbol{\beta}$ into covariance in (15), as follows:

$$
\widehat{\Sigma}(t)=\widehat{\Sigma}_{1}^{-1}(t) \widehat{\Sigma}_{2}(t) \widehat{\Sigma}_{1}^{-1}(t)
$$

where

$$
\begin{aligned}
& \widehat{\Sigma}_{1}(t)=n^{-1} \sum_{i=1}^{n} \int_{0}^{\tau} K_{h}(u-t)(\boldsymbol{u}-\boldsymbol{t})^{\otimes 2} I\left(C_{i} \geq u\right) V_{1}(u, \widehat{\boldsymbol{\beta}}) d \widetilde{N}_{i}(u) \\
& \widehat{\Sigma}_{2}(t)=n^{-1} \sum_{i=1}^{n} \int_{0}^{\tau} h K_{h}^{2}(u-t)(\boldsymbol{u}-\boldsymbol{t})^{\otimes 2} I\left(C_{i} \geq u\right) V_{2}(u, \widehat{\boldsymbol{\beta}}) \widehat{\mu}_{0}^{2}(u, \widehat{\beta}(u)) \exp \left(2 \widehat{\boldsymbol{\beta}}^{\mathrm{T}} z_{i}(\boldsymbol{u})\right) o_{i}(u) d u
\end{aligned}
$$

with

$$
\begin{aligned}
& V_{1}(u, \widehat{\boldsymbol{\beta}})=S_{n, 2}(u, \widehat{\boldsymbol{\beta}}) / S_{n, 0}(u, \widehat{\boldsymbol{\beta}})-\left\{S_{n, 1}(u, \widehat{\boldsymbol{\beta}}) / S_{n, 0}(u, \widehat{\boldsymbol{\beta}})\right\}^{2}, \\
& V_{2}(u, \widehat{\boldsymbol{\beta}})=\left\{z_{i}-S_{n, 1}(u, \widehat{\boldsymbol{\beta}}) / S_{n, 0}(u, \widehat{\boldsymbol{\beta}})\right\}^{2}, \\
& S_{n, j}(u, \widehat{\boldsymbol{\beta}})=n^{-1} \sum_{i=1}^{n} I\left(C_{i} \geq u\right) \exp \left(\widehat{\boldsymbol{\beta}}^{\mathrm{T}} z_{i}(\boldsymbol{u})\right) o_{i}(u) z_{i}^{j}, \quad j=0,1,2 .
\end{aligned}
$$

We show that $\widehat{\Sigma}_{1}(t)$ and $\widehat{\Sigma}_{2}(t)$ converges in probability to $\Sigma_{1}(t)$ and $\Sigma_{2}(t)$ presented in Appendix 2, respectively. Therefore the estimator $\widehat{\Sigma}(t)$ of the asymptotic covariance $\Sigma(t)=\sigma_{1}^{-2}(t) \sigma_{2}(t) \Omega_{1}^{-1} \Omega_{2} \Omega_{1}^{-1}$ in (15) is consistent. And detailed proofs are displayed in Appendix 2. Moreover, the finite sample performance of the variance estimation is validated in simulation studies.

\subsection{Asymptotic properties of baseline mean function}

As introduced in $\S 2 \cdot 2$, we use Breslow type estimator to evaluate the baseline mean function at each fixed time point. Here, we discuss the asymptotic properties of the estimator $\widehat{\mu}_{0}(t, \widehat{\beta}(t))$.

THEOREM 3. Under Conditions 1-5, assume that the bandwidth $h$ satisfies the conditions:

$$
h \rightarrow 0, \quad n h \rightarrow \infty, \quad \text { and } \quad n h^{5}=o(1),
$$

then the asymptotic distribution of $\widehat{\mu}_{0}(t, \widehat{\beta}(t))$ satisfies

$$
(n h)^{1 / 2}\left(\widehat{\mu}_{0}(t, \widehat{\beta}(t))-\mu_{0}(t)\right) \rightarrow N\left(0, \Sigma_{3}(t)\right),
$$

where $\Sigma_{3}(t)=\nu_{0} q_{0}^{-2}(t) q_{1}^{2}(t) \sigma_{1}^{-2}(t) \sigma_{2}(t)$, and $\nu_{0}=\int K^{2}(u) d u$.

The detailed proofs are presented in Appendix 2. Furthermore, the rate of convergence for $\widehat{\mu}_{0}(t, \widehat{\beta}(t))$ is $(n h)^{1 / 2}$ which is the same as the rate of $\widehat{\beta}(t)$. And the finite sample performance of the estimator is displayed in simulation studies.

\section{Simulation}

In this section, we evaluated the finite sample performance of the proposed local kernel estimator through a numerical study. In each simulated data set, we generated $n$ independent and identically distributed random variables $\left\{K_{i}, T_{i}, N_{i}, Z_{i}\right\}$. For each individual $i$, the number of observation $K_{i}$ was generated as a discrete uniform distribution on $\{1,2, \ldots, C\}$, where the number $C$ was finite. And the follow-up time $T_{i}=\left(T_{i 1}, \ldots, T_{i K_{i}}\right)$ were generated as an exponential distribution. The covariate $Z_{i}$ was generated from uniform distribution $U(0,1)$. Given the time-varying coefficient $\beta(t)$, we generated the 
recurrent event $N_{i}$ from nonhomogeneous Poisson process with mean function $\mu_{0}(t) \exp \left(\beta(t) z_{i}\right)$. That is, the event number between two consecutive observation times were generated from Poisson distribution with the mean $\mu_{0}\left(T_{i, j}\right) \exp \left(\beta\left(T_{i, j}\right) z_{i}\right)-\mu_{0}\left(T_{i, j-1}\right) \exp \left(\beta\left(T_{i, j-1}\right) z_{i}\right)$ and

$$
N_{i, j}-N_{i, j-1} \sim \text { Poisson }\left(\mu_{0}\left(T_{i, j}\right) \exp \left(\beta\left(T_{i, j}\right) z_{i}\right)-\mu_{0}\left(T_{i, j-1}\right) \exp \left(\beta\left(T_{i, j-1}\right) z_{i}\right)\right) .
$$

We considered the mean function model under two parameter settings. For each setting, we set $p=1$ and used Epanechnikov kernel to estimate the local kernel estimator with bandwidth $h$ equal to $0 \cdot 3$ and $0 \cdot 5$, respectively. We performed the simulation with sample sizes 300 and 500. For each setting, we generated 1000 datasets. In this section, we only showed the results under sample size of 300 , and the simulation results with sample size of 500 was presented in Appendix 3. We performed the estimation at 100 equally spaced grid points on the time interval. The maximum number of observed times for per individual was $C=10$, and the maximum follow-up time was 6 .

In the first setting, we set the regression function as $\beta(t)=\sqrt{ } t$, and the baseline function $\mu_{0}(t)=$ $2 t^{2}+2$. The results were shown in Figure 1. Panels a1 and a2 of Figure 1 presented the true curve $\beta(t)$, and the average of the local kernel estimator $\widehat{\beta}(t)$ with the bandwidth set at $0 \cdot 3$ and $0 \cdot 5$, respectively. The estimators were generally very close to the true value with a slight deviation on the boundary. Panels a3 and a 4 of Figure 1 compared the estimated and empirical standard errors of the local kernel estimator with bandwidth $0 \cdot 3$ and $0 \cdot 5$, respectively. As can be seen, there were good agreement between the estimated and empirical standard errors from different bandwidth, with slight bias near the boundary. Panels a5 and a6 of Figure 1 showed the empirical coverage probabilities of the $95 \%$ confidence intervals with bandwidth 0.3 and 0.5 , respectively. The empirical coverage probabilities were generally around $95 \%$ with lower coverage probabilities on the boundary due to the relative larger bias of the coefficient estimator. The simulation results with sample size of 500 showed similar pattern and were displayed in Appendix 3. Panels a7 and a8 of Figure 1 showed the baseline function estimator. The estimators were close to the true curve. The estimated curve of sample size 500 was closer to the true curve than that of sample size 300 slightly.

In the second setting, we set regression function as $\beta(t)=0 \cdot 5(\operatorname{Beta}(t / 12,3,3)+\operatorname{Beta}(t / 12,4,4))$, where $\operatorname{Beta}(\cdot)$ was the Beta density function, and the baseline function $\mu_{0}(t)=2+2 \sqrt{ } t$. Similar to the first setting, the results also had good performance as showed in Figure 2. Panels b1 and b2 of Figure 2 showed that the true curve $\beta(t)$, and the average of the local kernel estimator $\widehat{\beta}(t)$ with the bandwidth set at $0 \cdot 3$ and 0.5 , respectively. The estimators were very close to the true value with a slight bias near the boundary. Panels b3 and b4 of Figure 2 compared the estimated and empirical standard errors of the local kernel estimator with bandwidth equal to 0.3 and 0.5 , respectively. Obviously, there were good concordance between estimated and empirical standard errors, with slight deviation on the boundary. Panels b5 and b6 of Figure 2 displayed the empirical coverage probabilities of the $95 \%$ confidence intervals with bandwidth 0.3 and 0.5 , respectively. The empirical coverage probabilities were generally around $95 \%$. There were lower coverage probabilities on the boundary owing to the relative larger bias of the coefficient estimator. The simulation results with sample size of 500 showed analogous pattern and were presented in Appendix 3. Panels b7 and b8 of Figure 2 presented the baseline function estimator. The estimators were close to the true baseline curve. Same as the first setting, the estimated curve of sample size 500 was closer to the true curve than that of sample size 300 slightly.

In summary, the local kernel estimators performed well in terms of small estimation bias and good coverage probabilities of the confidence intervals. We will apply the estimation procedure to analyze a childhood asthma study data.

\section{APPLICATION}

The childhood wheezing study was designed and conducted at Indiana University School of Medicine (Tepper et al. (2008)). In this study, 105 infants with high risk of developing asthma were recruited. The cumulative wheezing episodes were collected by monthly phone call. The median follow-up time was 33.5 months, and the total number of wheezing events was 625 . For the baseline characteristics, $49.5 \%$ 
(a1)

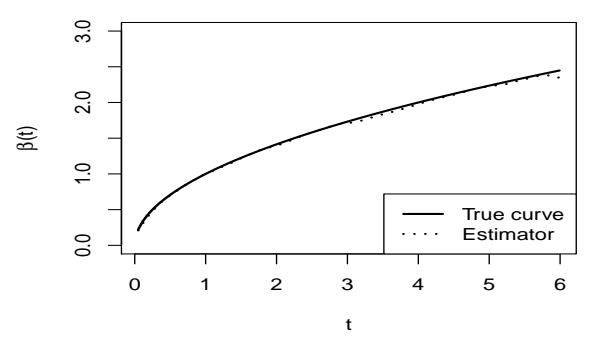

(a3)

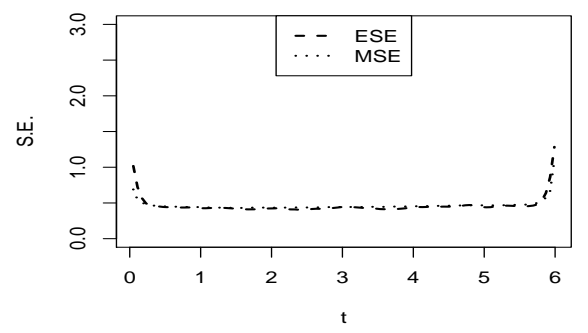

(a5)

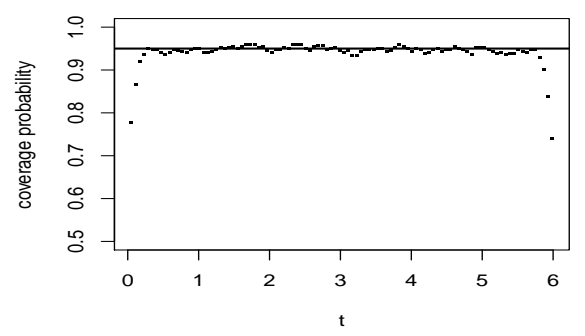

(a7)

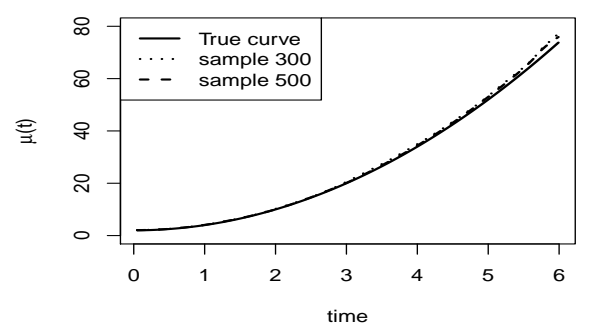

(a2)

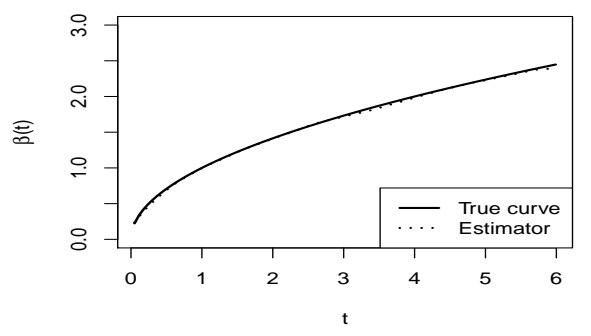

(a4)

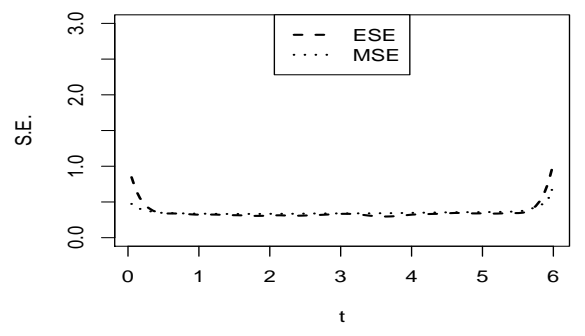

(a6)

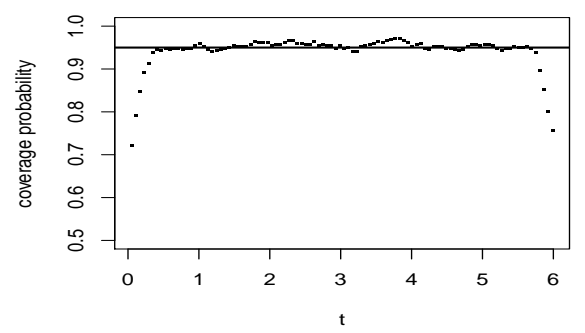

(a8)

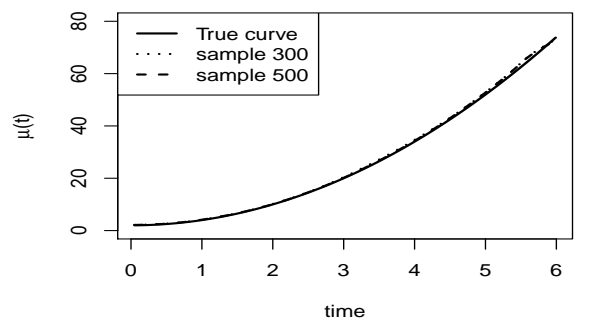

Fig. 1: (a1) and (a2): The true and the average of the local kernel estimator with bandwidth $0 \cdot 3$ and $0 \cdot 5$, respectively. (a3) and (a4): Comparison of empirical standard errors (ESE) and the estimated standard errors (MSE) of $\widehat{\beta}(t)$ with bandwidth $0 \cdot 3$ and $0 \cdot 5$, respectively; (a5) and (a6): Empirical coverage probabilities of the $95 \%$ confidence intervals for $\widehat{\beta}(t)$ with bandwidth $0 \cdot 3$ and $0 \cdot 5$, respectively. (a7) and (a8): Compare the true baseline curve and the average of the estimator with bandwidth $0 \cdot 3$ and $0 \cdot 5$, respectively, under sample sizes 300 and 500 . 
(b1)

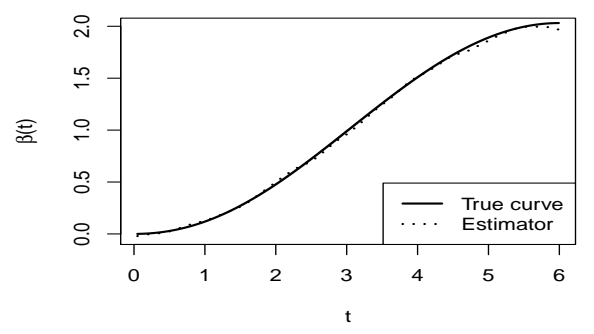

(b3)

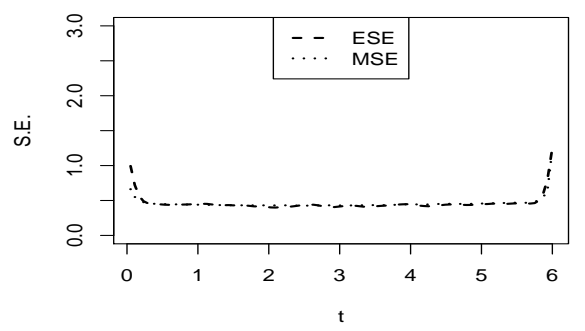

(b5)

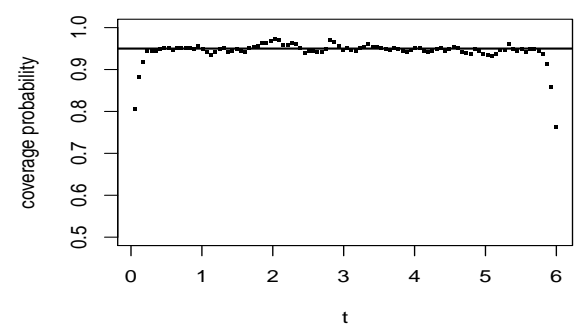

(b7)

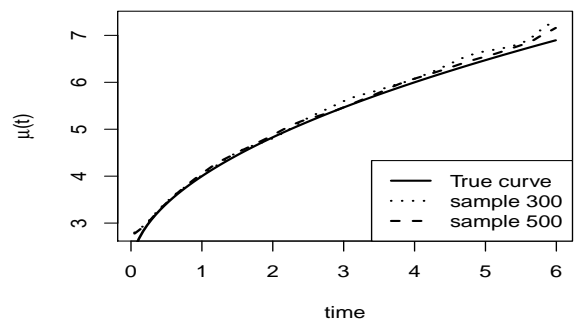

(b2)

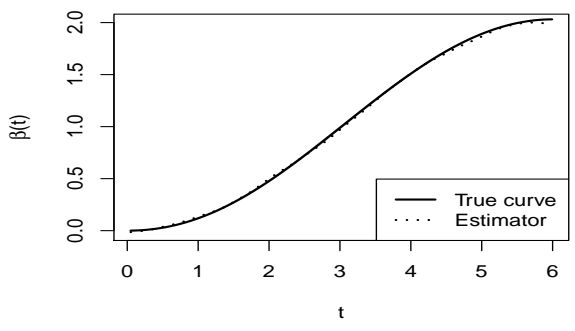

(b4)

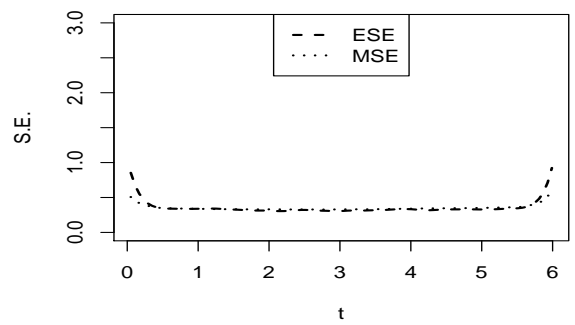

(b6)

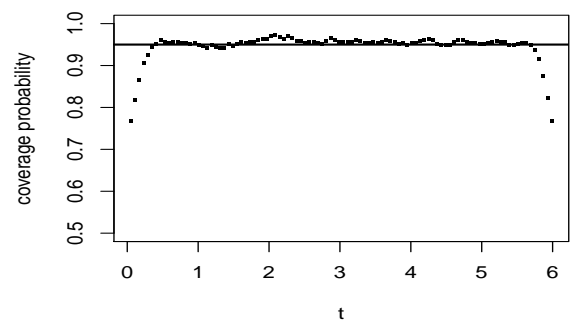

(b8)

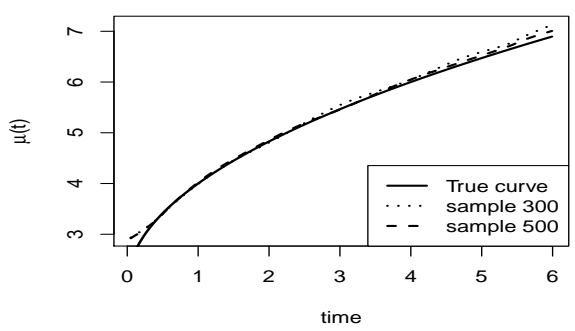

Fig. 2: (b1) and (b2): The true and the average of the local kernel estimator with bandwidth $0 \cdot 3$ and $0 \cdot 5$, respectively. (b3) and (b4): Comparison of empirical standard errors (ESE) and the estimated standard errors (MSE) of $\widehat{\beta}(t)$ with bandwidth 0.3 and 0.5 , respectively; (b5) and (b6): Empirical coverage probabilities of the $95 \%$ confidence intervals for $\widehat{\beta}(t)$ with bandwidth $0 \cdot 3$ and $0 \cdot 5$, respectively. (b7) and (b8): Compare the true baseline curve and the average of the estimator with bandwidth 0.3 and 0.5 , respectively, under sample sizes 300 and 500. 


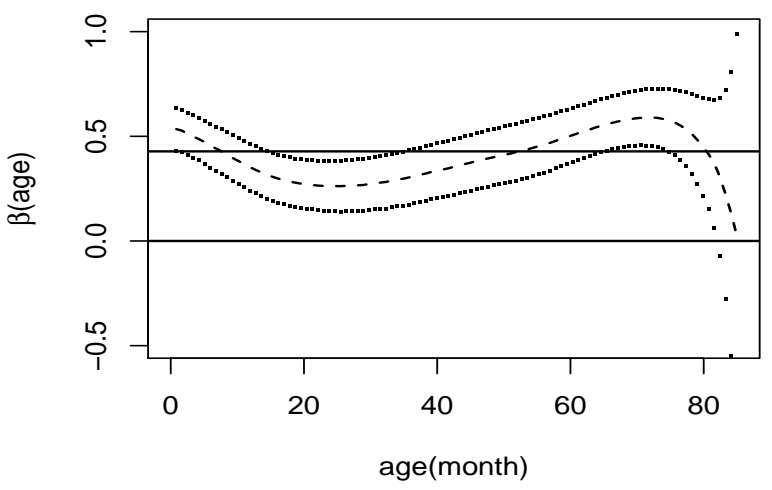

Fig. 3: Estimated IL-10 effect, $\widehat{\beta}$ (age), time-varying effect (dash); $95 \%$ confidence interval (dotted); IL-10 effect based on the model with a constant coefficient (horizontal solid line, $\beta=0.428$ ).

was boys, and $10.5 \%$ of children's mothers smoked during pregnancy. And the mean age at enrollment was $10 \cdot 8$ months. In recent human asthma study, Kearley et al. (2005) indicated that interleukin-10 (IL-10) regulated the suppressive activity of $\mathrm{T}$ cells, which played an important role in human asthma. Furthermore, Groux et al. (1998) showed that IL-10 had differential effects on T cells relying on their activated state. The potent anti-inflammatory cytokine IL-10 was shown to be risk factor for infection in early childhood (Yao et al. (2010)). Also the IL-10's effect may vary during the childhood growth. Therefore, we applied the proposed method to analyze the time-varying effect of interleukin IL-10 about the childhood wheeze data set.

We estimated the time-varying effect IL-10 on the risk of wheezing using the proposed local kernel estimator. The bandwidth was set as 20 and results were shown in Figure 3. In general, IL-10 had significant effect on the risk of child wheezing over the follow-up period. The relative risk increased over time period from 25 to 70 months and decreased over time near the boundary. We also estimated the IL-10 effect as a constant coefficient and the overall relative risk was 1.53 ( $p$-value $<0 \cdot 05)$. Although both timevarying and constant effect estimators showed significant results, the time-varying estimator demonstrated an increasing IL-10 effect as age increased. Overall, we illustrated IL-10 was positive associate with child wheezing. And subjects with an increased value of IL-10 will have higher wheezing risk.

\section{Discussion}

In this paper, we propose an local kernel estimation procedure for the panel count model with timevarying coefficients. We construct a kernel-weighted local partial likelihood at each fixed time point on the basis of local polynomial interpolation. The strong uniform consistency of the proposed estimator is derived. We also show that the proposed estimator is asymptotically normal under some regularity conditions. Furthermore, the simulation results demonstrate the proposed estimation methods perform well under finite sample sizes. The application of the proposed methods for the clinical data analysis also demonstrates that the time-varying coefficient estimation provides more information on the effect of risk factors on the panel count outcome measurement. Through this paper, we provide a nonparametric approach for time-varying coefficient in panel count data. Compared with the spline estimator for panel count model of which the asymptotic normality of $\widehat{\boldsymbol{\beta}}$ is not verified, our approach provides a thorough theoretical investigation. The inference of $\widehat{\boldsymbol{\beta}}$ is also developed. 
Meanwhile, there are challenges remaining in the local kernel method for panel count data, for example, the bandwidth selection. From (18), the theoretical optimal bandwidth depends on unknown quantities $\sigma_{1}(t), \sigma_{2}(t)$ and $\beta^{\prime \prime}(t)$. It turned out that bandwidth selection is hard problem for the panel count data model with nonparametric time-varying coefficients, which is beyond the scope of this paper. Nevertheless, there is warrant to select the optimal bandwidth based on data-driven technique. The common selection tool is cross validation method. Hoover et al. (1998) developed a cross validation criterion of bandwidth selection for longitudinal data. Cai et al. (2000) proposed a cross validation technique to select optimal bandwidth for time-varying model. Tian et al. (2005) discussed a $K$-fold cross validation method to select bandwidth for survival data. Therefore, developing a cross validation technique for time-varying coefficients panel count model is of future research interest.

\section{ACKNOWLEDGEMENT}

The research was supported in part by National Natural Science Foundation of China (11671256 Yu), and by Chinese Ministry of Science and Technology (2016YFC0902403 Yu), and by the University of Michigan and Shanghai Jiao Tong University Collaboration Grant (2017, Yu).

\section{APPENDIX 1}

\section{Notation and Lemmas}

In Appendices, the notations are the same as $\S 2$ and $\S 3$. For simplicity of presentation, we introduce some additional notations, let $\boldsymbol{\alpha}=H\left(\boldsymbol{\beta}-\boldsymbol{\beta}^{*}\right)=\left(\alpha_{0}, \alpha_{1}, \ldots, \alpha_{p}\right)^{\mathrm{T}}$, where $\alpha_{k}=h^{k}\left(\beta_{k}(t)-\right.$ $\left.\beta^{(k)}(t) / k !\right), H$ is $p$ th-order diagonal matrix and $\boldsymbol{\beta}^{*}$ is the true vector. $\tilde{z}_{i}(\boldsymbol{u})=H^{-1} z_{i}(\boldsymbol{u})=z_{i}(1,(u-$ $\left.t) / h, \ldots,(u-t)^{p} / h^{p}\right)^{\mathrm{T}}$. For a matrix $A=\left(a_{i j}\right),\|A\|=\sup _{i, j}\left|a_{i j}\right|$. For a vector $\boldsymbol{a},\|\boldsymbol{a}\|=\sup _{i}\left|a_{i}\right|$, and $|\boldsymbol{a}|=\left(\sum a_{i}^{2}\right)^{1 / 2}$. Some further definitions are:

For $j=0,1,2$, set

$$
\begin{aligned}
& S_{n, j}(u, \boldsymbol{\alpha})=n^{-1} \sum_{i=1}^{n} I\left(C_{i} \geq u\right) \exp \left(\boldsymbol{\alpha}^{\mathrm{T}} z_{i}(\boldsymbol{u})+\boldsymbol{\beta}^{* \mathrm{~T}} z_{i}(\boldsymbol{u})\right) o_{i}(u) z_{i}^{j}, \\
& S_{j}(u, \boldsymbol{\alpha})=E\left(p_{1}(u \mid z) p_{2}(u \mid z) \exp \left(\boldsymbol{\alpha}^{\mathrm{T}} \tilde{z}(\boldsymbol{u})+\boldsymbol{\beta}^{* \mathrm{~T}} z(\boldsymbol{u})\right) z^{j}\right)
\end{aligned}
$$

For $j=0,1,2$, set

$$
\begin{aligned}
& \widetilde{S}_{n, j}(u)=n^{-1} \sum_{i=1}^{n} I\left(C_{i} \geq u\right) \exp \left(\boldsymbol{\beta}^{* \mathrm{~T}} z_{i}(\boldsymbol{u})\right) o_{i}(u) \tilde{z}_{i}(\boldsymbol{u})^{\otimes j}, \\
& \widetilde{S}_{j}(u)=E\left(p_{1}(u \mid z) p_{2}(u \mid z) \exp \left(\boldsymbol{\beta}^{* \mathrm{~T}} z_{i}(\boldsymbol{u})\right) \tilde{z}(\boldsymbol{u})^{\otimes j}\right)
\end{aligned}
$$

For $j=0,1$, put

$$
\begin{aligned}
& \widetilde{S}_{n, j}^{*}(u)=n^{-1} \sum_{i=1}^{n} I\left(C_{i} \geq u\right) \exp \left(\beta(u) z_{i}\right) o_{i}(u) \tilde{z}_{i}^{j}(\boldsymbol{u}), \\
& \widetilde{S}_{j}^{*}(u)=E\left(p_{1}(u \mid z) p_{2}(u \mid z) \exp (\beta(u) z) \tilde{z}^{j}(\boldsymbol{u})\right)
\end{aligned}
$$

For $j=0,1,2$, set

$$
\begin{aligned}
& S_{n, j}\left(u, \boldsymbol{\beta}^{*}\right)=n^{-1} \sum_{i=1}^{n} I\left(C_{i} \geq u\right) \exp \left(\boldsymbol{\beta}^{* \mathrm{~T}} z_{i}(\boldsymbol{u})\right) o_{i}(u) z_{i}^{j}, \\
& S_{j}\left(u, \boldsymbol{\beta}^{*}\right)=E\left(p_{1}(u \mid z) p_{2}(u \mid z) \exp \left(\boldsymbol{\beta}^{* \mathrm{~T}} z_{i}(\boldsymbol{u})\right) z^{j}\right)
\end{aligned}
$$


For $j=0,1,2$, put

$$
\begin{aligned}
& S_{n, j}^{*}(u, \beta(u))=n^{-1} \sum_{i=1}^{n} I\left(C_{i} \geq u\right) \exp \left(\beta(u) z_{i}\right) o_{i}(u) z_{i}^{j}, \\
& S_{j}^{*}(u, \beta(u))=E\left(p_{1}(u \mid z) p_{2}(u \mid z) \exp (\beta(u) z) z^{j}\right) .
\end{aligned}
$$

LEMMA A1. Let

$$
c_{n}(u)=n^{-1} \sum_{i=1}^{n} I\left(C_{i} \geq u\right) o_{i}(u) g\left(u, z_{i}\right) \quad \text { and } \quad c(u)=E\left(p_{1}(u \mid z) p_{2}(u \mid z) g(u, z)\right),
$$

if $g\left(u, z_{i}\right)$ is bounded variation, then

$$
\sup _{u \in T}\left\|c_{n}(u)-c(u)\right\|=O_{p}\left(n^{-1 / 2}\right)
$$

Proof. Given $g\left(u, z_{i}\right)$ is bounded variation, and under Conditions 2 and 4 , we have $o_{i}(u) g\left(u, z_{i}\right)$ is bounded variation, then we can write $o_{i}(u) g\left(u, z_{i}\right)=g_{1}\left(u, z_{i}\right)-g_{2}\left(u, z_{i}\right)$, where both $g_{1}\left(u, z_{i}\right)$ and $g_{2}\left(u, z_{i}\right)$ are nonnegative and nondecreasing. Thus

$$
c_{n}(u)=n^{-1} \sum_{i=1}^{n}\left\{I\left(C_{i} \geq u\right) g_{1}\left(u, z_{i}\right)-I\left(C_{i} \geq u\right) g_{2}\left(u, z_{i}\right)\right\},
$$

and $I\left(C_{i} \geq u\right)$, for each $i$, is non-increasing in $u$, then by lemma A.2 of Bilias et al. (1997), $\left\{I\left(C_{i} \geq\right.\right.$ $u), u \in T\},\left\{g_{j}\left(u, z_{i}\right), u \in T\right\}_{j=1,2}$ have pseudodimension at most 1. By lemma 5.1 of Pollard (1990) combined with (A2), $\left\{I\left(C_{i} \geq u\right) o_{i}(u) g\left(u, z_{i}\right), u \in T\right\}$ has pseduodimension at most 10. Therefore, it must be Euclidean and certainly manageable according to theorem 4.8 of Pollard (1990). In view of Condition 2, we choose envelops as $B_{1} / \sqrt{ } n$, for some constant $B_{1}$. Then by theorem 8.3 (the uniform laws of large numbers) of Pollard (1990), we have $\sup _{u \in T}\left\|c_{n}(u)-c(u)\right\|=O_{p}\left(n^{-1 / 2}\right)$.

Lemma A2. Let $T=[a, b] \subset R$, suppose that

$$
\lim _{n \rightarrow \infty} \sup _{s \in T}\left\{\left|h_{n}(s)-h(s)\right|+\left|J_{n}(s)-J(s)\right|\right\}=0,
$$

where $h_{n}(\cdot), h(\cdot)$ are continuous on $T$, and $J_{n}(\cdot), J(\cdot)$ are right continuous with bounded variations on T. Then

$$
\begin{aligned}
& \lim _{n \rightarrow \infty} \sup _{s \in T}\left\{\left|\int_{a}^{s} h_{n}(u) J_{n}(d u)-\int_{a}^{s} h(u) J(d u)\right|\right\}=0, \\
& \lim _{n \rightarrow \infty} \sup _{s \in T}\left\{\left|\int_{a}^{s} h_{n}(u) J_{n}(d u)-\int_{a}^{s} h_{n}(u) J(d u)\right|\right\}=0 .
\end{aligned}
$$

Proof. First, since $h_{n}$ uniform converges to $h$, and $J_{n}, J$ are bounded variation functions with total variations bounded $B_{2}$, for some constant $B_{2}$. Then

$$
\begin{aligned}
& \lim _{n \rightarrow \infty} \sup _{s \in T}\left\{\left|\int_{a}^{s} h_{n}(u) J_{n}(d u)-\int_{a}^{s} h(u) J_{n}(d u)\right|\right\}=0, \\
& \lim _{n \rightarrow \infty} \sup _{s \in T}\left\{\left|\int_{a}^{s} h_{n}(u) J(d u)-\int_{a}^{s} h(u) J(d u)\right|\right\}=0 .
\end{aligned}
$$

Since

$$
\begin{aligned}
& \left|\int_{a}^{s} h_{n}(u) J_{n}(d u)-\int_{a}^{s} h_{n}(u) J(d u)\right| \\
& \leq\left|\int_{a}^{s} h_{n}(u) J_{n}(d u)-\int_{a}^{s} h(u) J(d u)\right|+\left|\int_{a}^{s} h(u) J(d u)-\int_{a}^{s} h_{n}(u) J(d u)\right| .
\end{aligned}
$$


Thus, from (A7) and (A8), we know that (A4) implies (A5). And since

$$
\begin{aligned}
& \left|\int_{a}^{s} h_{n}(u) J_{n}(d u)-\int_{a}^{s} h(u) J(d u)\right| \\
& \leq\left|\int_{a}^{s} h_{n}(u) J_{n}(d u)-\int_{a}^{s} h(u) J_{n}(d u)\right|+\left|\int_{a}^{s} h(u) J_{n}(d u)-\int_{a}^{s} h(u) J(d u)\right| .
\end{aligned}
$$

For the second term of the right-hand side in (A9), since $h(\cdot)$ is continuous, we can partition $T$ by $a=$ $s_{0}<\ldots<s_{n_{0}}=b$, and take constant $h_{j}\left(=h\left(s_{j}\right)\right)$ such that the simple function:

$$
h_{\varepsilon}(s)=\sum_{j=0}^{n_{0}-1} h_{j} I\left(s \in\left[s_{j}, s_{j+1}\right)\right)
$$

satisfies

$$
\sup _{s \in T}\left|h_{\varepsilon}(s)-h(s)\right|<\varepsilon
$$

Thus

$$
\begin{aligned}
& \left|\int_{a}^{s} h(u) J_{n}(d u)-\int_{a}^{s} h(u) J(d u)\right| \\
& \leq\left|\int_{a}^{s}\left\{h(u)-h_{\varepsilon}(u)\right\} J_{n}(d u)\right|+\left|\int_{a}^{s} h_{\varepsilon}(u)\left\{J_{n}(d u)-J(d u)\right\}\right|+\left|\int_{a}^{s}\left\{h(u)-h_{\varepsilon}(u)\right\} J(d u)\right| \\
& \leq 2 \varepsilon B_{2}+\left|\int_{a}^{s} \sum_{j=0}^{n_{0}-1} h_{j} I\left(u \in\left[s_{j}-s_{j+1}\right)\right)\left\{J_{n}(d u)-J(d u)\right\}\right| \\
& =2 \varepsilon B_{2}+\left|\sum_{j=0}^{n_{0}-1} h_{j} \int_{s_{j}}^{s_{j+1}}\left\{J_{n}(d u)-J(d u)\right\}\right| \\
& \leq 2 \varepsilon B_{2}+\sum_{j=0}^{n_{0}-1}\left|h_{j}\right|\left|J_{n}\left(s_{j+1}\right)-J\left(s_{j+1}\right)-J_{n}\left(s_{j}\right)+J\left(s_{j}\right)\right| \\
& \leq 2 \varepsilon B_{2}+2 \sum_{j=0}^{n_{0}-1}\left|h_{j}\right| \sup _{s \in T}\left|J_{n}(s)-J(s)\right| \\
& \rightarrow 2 \varepsilon B_{2} \quad \text { as } n \rightarrow \infty .
\end{aligned}
$$

This in conjunction with (A6) and (A9), we obtain (A4). And from (A4) and (A7), then (A5) holds.

\section{APPENDIX 2}

\section{Detailed techniques for theorem proofs}

Proof of Theorem 1. This proof is basically same as the proof of Lemma 2.2 of Hardle et al. (1988) and Theorem 2.1 of Zhao (1994). The major difference is that we have to treat a vector parameter $\boldsymbol{\beta}^{*}=$ $\left(\beta(t), \beta^{\prime}(t), \ldots, \beta^{(p)} / p !\right)^{\mathrm{T}}$ due to the local polynomial estimation. Next up, we will show detailed proof procedure by the below two lemmas. Introduce some notations as follows:

$$
G_{\alpha_{k} n 1}(t, t+s)=n^{-1} \sum_{i=1}^{n} \int_{0}^{\tau} I\left(C_{i} \geq u\right) I(t<u<t+s)((u-t) / h)^{k} z_{i} d \widetilde{N}_{i}(u)
$$

and

$$
G_{\alpha_{k} 1}(t, t+s)=E\left(G_{\alpha_{k} n 1}(t, t+s)\right)
$$




$$
\begin{aligned}
& G_{\alpha_{k} n 2}(t, t+s)=n^{-1} \sum_{i=1}^{n} \int_{0}^{\tau} I\left(C_{i} \geq u\right) I(t<u<t+s)((u-t) / h)^{k}\left(S_{n, 1}(u, \boldsymbol{\alpha}) / S_{n, 0}(u, \boldsymbol{\alpha})\right) \\
& d \widetilde{N}_{i}(u),
\end{aligned}
$$

and

$$
G_{\alpha_{k} 2}(t, t+s)=E\left(G_{\alpha_{k} n 2}(t, t+s)\right)
$$

and for $c>0$,

$$
\begin{aligned}
& V_{\alpha_{k} n 1}(t, c)=\sup _{|s| \leq c}\left|G_{\alpha_{k} n 1}(t, t+s)-G_{\alpha_{k} 1}(t, t+s)\right|, \\
& V_{\alpha_{k} n 2}(t, c)=\sup _{|s| \leq c}\left|G_{\alpha_{k} n 2}(t, t+s)-G_{\alpha_{k} 2}(t, t+s)\right|,
\end{aligned}
$$

where $S_{n, j}(u, \boldsymbol{\alpha}), j=0,1$ defined in Appendix 1, and $\alpha_{k}$ is the $k$ th component of $\boldsymbol{\alpha}$. Note that $\sup \left\{\alpha_{k}\right\}=\bar{\alpha}_{k}$, and $\inf \left\{\alpha_{k}\right\}=\underline{\alpha}_{k}$.

LeMmA B1. Let $0<c_{n} \rightarrow 0$, as $n \rightarrow \infty$, and $1<c_{n}^{-1} \leq(n / \log n)^{1-2 / \lambda}$, then almost surely (a.s.),

$$
V_{n 1}=\sup _{t \in T} \sup _{\alpha_{k} \in \mathcal{N}_{0}} V_{\alpha_{k} n 1}\left(t, c_{n}\right)=O\left(n^{-1 / 2}\left(c_{n} \log n\right)^{1 / 2}\right), \quad \text { as } \quad n \rightarrow \infty,
$$

and

$$
V_{n 2}=\sup _{t \in T} \sup _{\alpha_{k} \in \mathcal{N}_{0}} V_{\alpha_{k} n 2}\left(t, c_{n}\right)=O\left(n^{-1 / 2}\left(c_{n} \log n\right)^{1 / 2}\right), \quad \text { as } \quad n \rightarrow \infty .
$$

where $\mathcal{N}_{0}:=\left\{\alpha_{k}:\left|\alpha_{k}-0\right|<\epsilon\right\}$.

Proof. Since $V_{\alpha_{k} n 1}$ is a special case of $V_{\alpha_{k} n 2}$, when substituted $S_{n, 1}(u, \boldsymbol{\alpha}) / S_{n, 0}(u, \boldsymbol{\alpha})$ by $z_{i}$. We only need to prove (B6). Put

$$
a_{n}=n^{-1 / 2}\left(c_{n} \log n\right)^{1 / 2}
$$

As we can treat the positive and negative part of $z_{i}$, separately, we assume that $z_{i}$ is nonnegative. First, we reduce $\sup _{\alpha_{k} \in \mathcal{N}_{0}}$ in (B6) to a maximum on a finite set. We use finite points $b_{1}<b_{2}<\ldots<b_{N_{n}}$ to partition $\mathcal{N}_{0}$, such that $b_{1}-\underline{\alpha}_{k} \leq a_{n}, \bar{\alpha}_{k}-b_{N_{n}} \leq a_{n}$, and $b_{j}-b_{j-1} \leq a_{n}$, for $2 \leq j \leq N_{n}$. Further, we assume that

$$
N_{n} \leq 2\left(\bar{\alpha}_{k}-\underline{\alpha}_{k}\right) / a_{n}
$$

and for any $t \in T$, and $|s| \leq c_{n}$, by Cauchy-Schwarz inequality, the functions $G_{\alpha_{k} n 2}(t, t+s)$ and $G_{\alpha_{k} 2}(t, t+s)$ are monotone in $\alpha_{k}$. Letting $J_{n}$ denote the set $\left\{\underline{\alpha}_{k}, b_{1}, \ldots, b_{N_{n}}, \bar{\alpha}_{k}\right\}$, and $J_{n}^{*}$ the set $\left\{\left(\underline{\alpha}_{k}, b_{1}\right),\left(b_{1}, b_{2}\right), \ldots,\left(b_{N_{n}}, \bar{\alpha}_{k}\right)\right\}$. Hence, we have, for any $\alpha_{k} \in \mathcal{N}_{0}$,

$$
\begin{aligned}
& G_{b_{k} n 2}(t, t+s)-G_{b_{k} 2}(t, t+s)+G_{b_{k} 2}(t, t+s)-G_{b_{k+1} 2}(t, t+s) \\
& \leq G_{\alpha_{k} n 2}(t, t+s)-G_{\alpha_{k} 2}(t, t+s) \\
& \leq G_{b_{k+1} n 2}(t, t+s)-G_{b_{k+1} 2}(t, t+s)+G_{b_{k+1} 2}(t, t+s)-G_{b_{k} 2}(t, t+s) .
\end{aligned}
$$

Thus

$$
\begin{aligned}
& \left|G_{\alpha_{k} n 2}(t, t+s)-G_{\alpha_{k} 2}(t, t+s)\right| \\
& \leq \max _{\alpha_{k} \in J_{n}}\left|G_{\alpha_{k} n 2}(t, t+s)-G_{\alpha_{k} 2}(t, t+s)\right|+\max _{\left(\alpha_{k}^{\prime}, \alpha_{k}^{\prime \prime}\right) \in J_{n}^{*}}\left|G_{\alpha_{k}^{\prime \prime} 2}(t, t+s)-G_{\alpha_{k}^{\prime} 2}(t, t+s)\right| .
\end{aligned}
$$


For $\alpha_{k}^{\prime}<\alpha_{k}^{\prime \prime}$,

$$
\begin{aligned}
& G_{\alpha_{k}^{\prime \prime} 2}(t, t+s)-G_{\alpha_{k}^{\prime} 2}(t, t+s) \mid \\
& =\mid \int_{0}^{\tau} I(t<u<t+s)((u-t) / h)^{k} S_{0}^{*}(u, \beta(u))\left\{S_{2}(u, \boldsymbol{\alpha}) / S_{0}(u, \boldsymbol{\alpha})-\left(S_{1}(u, \boldsymbol{\alpha}) / S_{0}(u, \boldsymbol{\alpha})\right)^{2}\right\}\left(\alpha_{k}^{\prime \prime}\right. \\
& \left.-\alpha_{k}^{\prime}\right) d u \mid \\
& \leq\left|\int_{0}^{\tau} I(t<u<t+s)((u-t) / h)^{k} M_{0}\left(\alpha_{k}^{\prime \prime}-\alpha_{k}^{\prime}\right) d u\right| \\
& \leq M_{0} a_{n},
\end{aligned}
$$

there exists some positive constant $M_{0}$ satisfied the upper inequality, under Conditions 1-5.

Hence

$$
V_{n 2} \leq \sup _{t \in T} \max _{\alpha_{k} \in J_{n}} V_{\alpha_{k} n 2}\left(t, c_{n}\right)+M_{0} a_{n} .
$$

Next, we reduce $\sup _{t \in T}$ to a maximum on a finite set. Now we partition $T$ by an equally-spaced grid $I_{n}:=\left\{t_{k}: t_{k}=k c_{n}, k=0, \ldots,\left[\tau / c_{n}\right]\right\}$, with $t_{\left[\tau / c_{n}\right]+1}=\tau$, where [.] denote the greatest integer part. For any $t \in T$ and $|s| \leq c_{n}$, there exists a grid point $t_{k}$, such that both $t$ and $t+s$ are between $t_{k}$ and $t_{k+1}$. And

$$
\begin{aligned}
& \left|G_{\alpha_{k} n 2}(t, t+s)-G_{\alpha_{k} 2}(t, t+s)\right| \\
& \leq\left|G_{\alpha_{k} n 2}\left(t_{k}, t+s\right)-G_{\alpha_{k} 2}\left(t_{k}, t+s\right)\right|+\left|G_{\alpha_{k} n 2}\left(t_{k}, t\right)-G_{\alpha_{k} 2}\left(t_{k}, t\right)\right| .
\end{aligned}
$$

Then, we obtain

$$
\left|G_{\alpha_{k} n 2}(t, t+s)-G_{\alpha_{k} 2}(t, t+s)\right| \leq 2 \max _{t \in I_{n}} V_{\alpha_{k} n 2}\left(t, c_{n}\right) .
$$

Thus

$$
V_{n 2} \leq 2 \max _{t \in I_{n}} \max _{\alpha_{k} \in J_{n}} V_{\alpha_{k} n 2}\left(t, c_{n}\right)+2 M_{0} a_{n} .
$$

In order to apply Bernstein's inequality, we truncate $\left\{z_{i}\right\}$ by some value, and define $V_{\alpha_{k} n 2}^{*}\left(t, c_{n}\right)$ similar to $V_{\alpha_{k} n 2}\left(t, c_{n}\right)$. Put

$$
Q_{n}=c_{n} / a_{n}
$$

and

$$
\begin{aligned}
& G_{\alpha_{k} n 2}^{*}(t, t+s)=n^{-1} \sum_{i=1}^{n} \int_{0}^{\tau} I\left(C_{i} \geq u\right)((u-t) / h)^{k} I(t<u<t+s)\left\{\sum _ { j = 1 } ^ { n } I ( C _ { j } \geq u ) \operatorname { e x p } \left(\boldsymbol{\alpha}^{\mathrm{T}} \tilde{z}_{j}(\boldsymbol{u})\right.\right. \\
& \left.\left.+\boldsymbol{\beta}^{* \mathrm{~T}} z_{j}(\boldsymbol{u})\right) z_{j} I\left(z_{j} \leq Q_{n}\right) o_{j}(u) / S_{n, 0}(u, \boldsymbol{\alpha})\right\} d \widetilde{N}_{i}(u),
\end{aligned}
$$

and

$$
G_{\alpha_{k} 2}^{*}(t, t+s)=E\left(G_{\alpha_{k} n 2}^{*}(t, t+s)\right) .
$$

Likewise, we have

$$
\begin{gathered}
V_{\alpha_{k} n 2}^{*}\left(t, c_{n}\right)=\sup _{|s| \leq c_{n}}\left|G_{\alpha_{k} n 2}^{*}(t, t+s)-G_{\alpha_{k} 2}^{*}(t, t+s)\right|, \\
V_{n 2}^{*}=\max _{t \in I_{n}} \max _{\alpha_{k} \in J_{n}} V_{\alpha_{k} n 2}^{*}\left(t, c_{n}\right) .
\end{gathered}
$$

Thus

$$
V_{n 2} \leq V_{n 2}^{*}+2 M_{0} a_{n}+2 A_{n 1}+2 A_{n 2}
$$


where

$$
\begin{gathered}
A_{n 1}=\sup _{t \in I_{n}} \sup _{\alpha_{k} \in J_{n}} \sup _{|s| \leq c_{n}}\left(G_{\alpha_{k} n 2}(t, t+s)-G_{\alpha_{k} n 2}^{*}(t, t+s)\right), \\
A_{n 2}=\sup _{t \in I_{n}} \sup _{\alpha_{k} \in J_{n}} \sup _{|s| \leq c_{n}}\left(G_{\alpha_{k} 2}(t, t+s)-G_{\alpha_{k} 2}^{*}(t, t+s)\right) .
\end{gathered}
$$

For

$$
\begin{aligned}
& \left.G_{\alpha_{k} n 2}(t, t+s)-G_{\alpha_{k} n 2}^{*}(t, t+s)\right) \\
& =n^{-1} \sum_{i=1}^{n} \int_{0}^{\tau} I\left(C_{i} \geq u\right)((u-t) / h)^{k} I(t<u<t+s)\left\{\sum_{j=1}^{n} I\left(C_{j} \geq u\right) \exp \left(\boldsymbol{\alpha}^{\mathrm{T}} \tilde{z}_{j}(\boldsymbol{u})+\boldsymbol{\beta}^{* \mathrm{~T}} z_{j}(\boldsymbol{u})\right)\right. \\
& \left.z_{j} I\left(z_{j}>Q_{n}\right) o_{j}(u) / S_{n, 0}(u, \boldsymbol{\alpha})\right\} d \widetilde{N}_{i}(u) \\
& \leq Q_{n}^{1-\lambda} n^{-1} \sum_{i=1}^{n} \int_{0}^{\tau} I\left(C_{i} \geq u\right)\left\{\sum_{j=1}^{n} I\left(C_{j} \geq u\right) \exp \left(\boldsymbol{\alpha}^{\mathrm{T}} \tilde{z}_{j}(\boldsymbol{u})+\boldsymbol{\beta}^{* \mathrm{~T}} z_{j}(\boldsymbol{u})\right) z_{j}^{\lambda} o_{j}(u) / S_{n, 0}(u, \boldsymbol{\alpha})\right\} \\
& d \widetilde{N}_{i}(u) .
\end{aligned}
$$

We have, by the classical strong low of large numbers and Lemma A1,

$$
\begin{aligned}
& n^{-1} \sum_{i=1}^{n} \int_{0}^{\tau} I\left(C_{i} \geq u\right)\left\{\sum_{j=1}^{n} I\left(C_{j} \geq u\right) \exp \left(\boldsymbol{\alpha}^{\mathrm{T}} \tilde{z}_{j}(\boldsymbol{u})+\boldsymbol{\beta}^{* \mathrm{~T}} z_{j}(\boldsymbol{u})\right) z_{j}^{\lambda} o_{j}(u) / S_{n, 0}(u, \boldsymbol{\alpha})\right\} d \widetilde{N}_{i}(u) \rightarrow \\
& \left.\int_{0}^{\tau} S_{0}^{*}(u, \beta(u)) E\left(p_{1}(u \mid z) p_{2}(u \mid z) \exp \left(\boldsymbol{\alpha}^{\mathrm{T}} \tilde{z}(\boldsymbol{u})+\boldsymbol{\beta}^{* \mathrm{~T}} z(\boldsymbol{u})\right) z^{\lambda}\right) / S_{0}(u, \boldsymbol{\alpha}) d u<\infty, \quad \text { a.s. } \quad \text { (B1 }\right)
\end{aligned}
$$

Noting that

$$
a_{n}^{-1} Q_{n}^{1-\lambda}=\left(c_{n}^{-1}(\log n / n)^{1-2 / \lambda}\right)^{\lambda / 2}=o(1) .
$$

From (B11), (B12) and (B13), we have, as $n \rightarrow \infty$,

$$
a_{n}^{-1} A_{n 1} \rightarrow 0, \quad \text { a.s. }
$$

From (B11), (B12), (B13) and $A_{n 2} \leq E\left(A_{n 1}\right)$, then, as $n \rightarrow \infty$,

$$
a_{n}^{-1} A_{n 2} \rightarrow 0, \quad \text { a.s. }
$$

Then, combining (B10), (B14) and (B15), it suffices for (B6) to show

$$
V_{n 2}^{*}=O\left(a_{n}\right) \text { a.s. }
$$

Next we will find a suitable upper bound for $\operatorname{pr}\left(V_{n 2}^{*} \geq B_{0} a_{n}\right)$ by appropriate choice of $B_{0}$. Now we perform a further partition for $V_{\alpha_{k} n 2}^{*}\left(t, c_{n}\right)$ at a fixed $t \in I_{n}$. Set $w_{n}=\left[\left(Q_{n} c_{n} / a_{n}\right)+1\right]$, and $s_{r}=r c_{n} / w_{n}$, for $r=-w_{n},-w_{n}+1, \ldots, w_{n}$. Since $G_{\alpha_{k} n 2}^{*}(t, t+s)$ and $G_{\alpha_{k} 2}^{*}(t, t+s)$ are monotone in $|s|$, suppose that $0 \leq s_{r} \leq s \leq s_{r+1}$, then

$$
\begin{aligned}
& G_{\alpha_{k} n 2}^{*}\left(t, t+s_{r}\right)-G_{\alpha_{k} 2}^{*}\left(t, t+s_{r}\right)+G_{\alpha_{k} 2}^{*}\left(t, t+s_{r}\right)-G_{\alpha_{k} 2}^{*}\left(t, t+s_{r+1}\right) \\
& \leq G_{\alpha_{k} n 2}^{*}(t, t+s)-G_{\alpha_{k} 2}^{*}(t, t+s) \\
& \leq G_{\alpha_{k} n 2}^{*}\left(t, t+s_{r+1}\right)-G_{\alpha_{k} 2}^{*}\left(t, t+s_{r+1}\right)+G_{\alpha_{k} 2}^{*}\left(t, t+s_{r+1}\right)-G_{\alpha_{k} 2}^{*}\left(t, t+s_{r}\right),
\end{aligned}
$$

from which we obtain

$$
\left|G_{\alpha_{k} n 2}^{*}(t, t+s)-G_{\alpha_{k} 2}^{*}(t, t+s)\right| \leq \max \left\{\xi_{n, r}, \xi_{n, r+1}\right\}+G_{\alpha_{k} 2}^{*}\left(t+s_{r}, t+s_{r+1}\right),
$$

where

$$
\xi_{n, r}=\left|G_{\alpha_{k} n 2}^{*}\left(t, t+s_{r}\right)-G_{\alpha_{k} 2}^{*}\left(t, t+s_{r}\right)\right| .
$$


The same holds for $s_{r} \leq s \leq s_{r+1} \leq 0$. Therefore

$$
V_{\alpha_{k} n 2}^{*}\left(t, c_{n}\right) \leq \max _{-w_{n} \leq r \leq w_{n}} \xi_{n, r}+\max _{-w_{n} \leq r \leq w_{n}-1} G_{\alpha_{k} 2}^{*}\left(t+s_{r}, t+s_{r+1}\right) .
$$

For all $r$, under Condition 5,

$$
G_{\alpha_{k} 2}^{*}\left(t+s_{r}, t+s_{r+1}\right) \leq \int_{t+s_{r}}^{t+s_{r+1}} q_{0}(u) Q_{n} d u \leq M_{3} Q_{n}\left(s_{r+1}-s_{r}\right) \leq M_{3} a_{n},
$$

so that

$$
\operatorname{pr}\left(V_{\alpha_{k} n 2}^{*}\left(t, c_{n}\right) \geq B_{0} a_{n}\right) \leq \operatorname{pr}\left(\max _{-w_{n} \leq r \leq w_{n}} \xi_{n, r} \geq\left(B_{0}-M_{3}\right) a_{n}\right)
$$

Now, let

$$
\begin{aligned}
& X_{i}=\int_{0}^{\tau} I\left(C_{i} \geq u\right)((u-t) / h)^{k} I(t<u<t+s)\left\{\sum _ { j = 1 } ^ { n } I ( C _ { j } \geq u ) \operatorname { e x p } ( \boldsymbol { \alpha } ^ { \mathrm { T } } \tilde { z } _ { j } ( \boldsymbol { u } ) + \boldsymbol { \beta } ^ { * \mathrm { T } } z _ { j } ( \boldsymbol { u } ) ) z _ { j } I \left(z_{j}\right.\right. \\
& \left.\left.\geq Q_{n}\right) o_{j}(u) / S_{n, 0}(u, \boldsymbol{\alpha})\right\} d \widetilde{N}_{i}(u)
\end{aligned}
$$

then

$$
\xi_{n r}=\left|n^{-1} \sum_{i=1}^{n}\left\{X_{i}-E\left(X_{i}\right)\right\}\right| .
$$

For

$$
\left|X_{i}-E\left(X_{i}\right)\right| \leq\left|\int_{0}^{\tau} I\left(C_{i} \geq u\right)((u-t) / h)^{k} I(t<u<t+s) Q_{n} d \widetilde{N}_{i}(u)\right| \leq \bar{N} Q_{n},
$$

where $\bar{N}=\tau \sup _{u \in T} N_{i}(u)$.

And, for some constant $M_{4}$, we have

$$
\begin{aligned}
& \sum_{i=1}^{n} \operatorname{var}\left(X_{i}\right) \leq \sum_{i=1}^{n} E\left(X_{i}^{2}\right) \\
& \leq \sum_{i=1}^{n} \int_{0}^{\tau} I\left(t \leq u \leq t+s_{r}\right)((u-t) / h)^{k} E\left(p_{1}(u \mid z) p_{2}(u \mid z) E\left(N^{2}(u) \mid z\right)\right)\left\{E \left(p_{1}(u \mid z) p_{2}(u \mid z)\right.\right. \\
& \left.\left.\exp \left(\boldsymbol{\alpha}^{\mathrm{T}} \tilde{z}(\boldsymbol{u})+\boldsymbol{\beta}^{* \mathrm{~T}} z(\boldsymbol{u})\right) z I\left(z \leq Q_{n}\right)\right) / S_{0}(u, \boldsymbol{\alpha})\right\}^{2} d u \\
& \leq \sum_{i=1}^{n} \int_{t+s_{r}}^{t+s_{r+1}} M_{4} d u \leq n M_{4} c_{n} .
\end{aligned}
$$

Then, by Bernstein's inequality,

$$
\begin{aligned}
\operatorname{pr}\left(\xi \geq\left(B_{0}-M_{3}\right) a_{n}\right) & \leq \exp \left\{-\left(\left(B_{0}-M_{3}\right) n a_{n}\right)^{2} / 2\left(\sum_{i=1}^{n} \operatorname{var}\left(X_{i}\right)+3^{-1}\left(B_{0}-M_{0}\right) \bar{N} Q_{n} n a_{n}\right)\right\} \\
& \leq \exp \left\{-\left(\left(B_{0}-M_{3}\right) n a_{n}\right)^{2} / 2\left(M_{4} n c_{n}+3^{-1}\left(B_{0}-M_{0}\right) \bar{N} Q_{n} n a_{n}\right)\right\} \leq n^{-B_{0}^{*}},
\end{aligned}
$$

where

$$
B_{0}^{*}=\left(B_{0}-M_{3}\right)^{2} / 2\left(M_{4}+3^{-1}\left(B_{0}-M_{3}\right) \bar{N}\right) .
$$

By (B18) and Boole's inequality,

$$
\operatorname{pr}\left(\sup _{t \in I_{n}} \sup _{\alpha_{k} \in J_{n}} V_{\alpha_{k} n 2}^{*}\left(t, c_{n}\right) \geq B_{0} a_{n}\right) \leq\left(N_{n}+2\right)\left(\left[\tau / c_{n}\right]+1\right) 2\left[\left(Q_{n} c_{n} / a_{n}\right)+1\right] n^{-B_{0}^{*}},
$$

From (B7), we obtain

$$
N_{n}+2 \leq 2\left(\bar{\alpha}_{k}-\underline{\alpha}_{k}\right) a_{n}^{-1}+2 .
$$


And, obviously,

$$
\left[\tau / c_{n}\right]+1 \leq(\tau+1) c_{n}^{-1}
$$

Also,

$$
2\left[\left(Q_{n} c_{n} / a_{n}\right)+1\right] \leq\left(2 Q_{n} c_{n} / a_{n}\right)+2 \leq 2\left(\left(c_{n} a_{n}^{-1}\right)^{2}+1\right),
$$

since

$$
\left(c_{n} a_{n}^{-1}\right)^{2}=c_{n} n / \log n \geq c_{n}^{-2 /(\lambda-2)} \geq 1
$$

then, we have,

$$
2\left[\left(Q_{n} c_{n} / a_{n}\right)+1\right] \leq 3 c_{n}^{2} a_{n}^{-2} .
$$

Hence

$$
\operatorname{pr}\left(V_{n 2}^{*} \geq B_{0} a_{n}\right) \leq 2\left(\bar{\alpha}_{k}-\underline{\alpha}_{k}+1\right)(\tau+1) 3 c_{n} a_{n}^{-3} n^{-B_{0}^{*}} \leq \bar{M}_{0}(n / \log n)^{(2 \lambda-1 / \lambda)} n^{-B_{0}^{*},}
$$

for some constant $\bar{M}_{0}$.

Given $\lambda$ and real $\kappa>0$, we choose a suitable $B_{0}$ denoted as $B_{\kappa, \lambda}$ to make the constant $B_{0}^{*}$ in (B19) satisfies

$$
B_{0}^{*} \geq \kappa+(2 \lambda-1) / \lambda
$$

And using $(2 \lambda-1) / \lambda=2-1 / \lambda>1$, for $\lambda>2$, then (B21) yields

$$
\operatorname{pr}\left(V_{n 2}^{*} \geq B_{\kappa, \lambda} a_{n}\right) \leq \bar{M}_{0}(\log n)^{-1} n^{-\kappa} .
$$

When $\kappa \geq 2$ in (B22), $\operatorname{pr}\left(V_{n 2}^{*} \geq B_{\kappa, \lambda} a_{n}\right)$ is summable in $n$. So, applying the Borel-Cantelli lemma,

$$
V_{n 2}^{*}=O\left(a_{n}\right), \quad \text { a.s. }
$$

Thus, form (B10), (B14), (B15) and (B23), we have

$$
V_{n 2}=O\left(a_{n}\right), \quad \text { a.s. }
$$

Similarly, we can also prove $V_{n 1}=O\left(a_{n}\right)$, a.s.

LEMmA B2. Let $h$ be a bandwidth and $c_{n}=2 h$. Assume that $h \rightarrow 0$ and $h^{-1}(\log n / n)^{1-2 / \lambda}=o(1)$, let

$$
U_{n k}(\boldsymbol{\alpha})=n^{-1} \sum_{i=1}^{n} \int_{0}^{\tau} I\left(C_{i} \geq u\right) K_{h}(u-t)((u-t) / h)^{k}\left\{z_{i}-S_{n, 1}(u, \boldsymbol{\alpha}) / S_{n, 0}(u, \boldsymbol{\alpha})\right\} d \widetilde{N}_{i}(u),
$$

Then we have

$$
\sup _{t \in T} \sup _{\alpha_{k} \in \mathcal{N}_{0}}(n h / \log n)^{1 / 2}\left|U_{n k}(\boldsymbol{\alpha})-E\left(U_{n k}(\boldsymbol{\alpha})\right)\right|=O(1), \quad \text { a.s. }
$$

Proof. Since $K(\cdot)$ is bounded variation function, so we can write $K(\cdot)=K_{1}(\cdot)-K_{2}(\cdot)$, where $K_{1}(\cdot)$ and $K_{2}(\cdot)$ are both increasing functions. Without loss of generality, suppose that $K_{1}(-1)=K_{2}(-1)=0$. Next up, we apply Lemma B1 by letting $c_{n}=2 h$. It is clear that the assumption of Lemma B1 hold here. Write

$$
\begin{aligned}
U_{n k}(\boldsymbol{\alpha})= & \int_{-h}^{h}\left\{n^{-1} \sum_{i=1}^{n} \int_{0}^{\tau} I\left(C_{i} \geq u\right) I(v<u-t<h)((u-t) / h)^{k}\left(z_{i}-S_{n, 1}(u, \boldsymbol{\alpha}) / S_{n, 0}(u, \boldsymbol{\alpha})\right)\right. \\
& \left.d \widetilde{N}_{i}(u)\right\} d K_{h}(v) \\
= & \int_{-h}^{h}\left\{G_{\alpha_{k} n 1}(t+v, t+h)-G_{\alpha_{k} n 2}(t+v, t+h)\right\} d K_{h}(v),
\end{aligned}
$$


where $G_{\alpha_{k} n 1}$ and $G_{\alpha_{k} n 2}$ defined as (B1) and (B2), respectively. So, we have

$$
\begin{aligned}
\sup _{t \in T} \sup _{\alpha_{k} \in \mathcal{N}_{0}}\left|U_{n k}(\boldsymbol{\alpha})-E\left(U_{n k}(\boldsymbol{\alpha})\right)\right| & \leq \sup _{t \in T} \sup _{\alpha_{k} \in \mathcal{N}_{0}}\left\{V_{\alpha_{k} n 1}(t, 2 h)+V_{\alpha_{k} n 2}(t, 2 h)\right\} \int_{-h}^{h} d K_{h}(v) \\
& \leq\left(K_{1}(1)+K_{2}(1)\right) h^{-1} \sup _{t \in T} \sup _{\alpha_{k} \in \mathcal{N}_{0}}\left\{V_{\alpha_{k} n 1}(t, 2 h)+V_{\alpha_{k} n 2}(t, 2 h)\right\} .
\end{aligned}
$$

Hence, by the consequence of Lemma B1, we can derive

$$
\sup _{t \in T} \sup _{\alpha_{k} \in \mathcal{N}_{0}}\left|U_{n k}(\boldsymbol{\alpha})-E\left(U_{n k}(\boldsymbol{\alpha})\right)\right|=O\left((\log n /(n h))^{1 / 2}\right), \quad \text { a.s. }
$$

Thus establishing (B25).

Next, we will prove Theorem 1. Since $\boldsymbol{\alpha}=H\left(\boldsymbol{\beta}-\boldsymbol{\beta}^{*}\right)$ and $\alpha_{k}(t)=\alpha_{k}=h^{k}\left(\beta_{k}(t)-\beta^{(k)}(t) / k !\right)$ defined in Appendix 1, from (8), we have

$$
\mathcal{L}_{n}(\boldsymbol{\alpha})=n^{-1} \sum_{i=1}^{n} \int_{0}^{\tau} I\left(C_{i} \geq u\right) K_{h}(u-t)\left\{\boldsymbol{\alpha}^{\mathrm{T}} \tilde{z}_{i}(\boldsymbol{u})+\boldsymbol{\beta}^{* \mathrm{~T}} z_{i}(\boldsymbol{u})-\log S_{n, 0}(u, \boldsymbol{\alpha})\right\} d \widetilde{N}_{i}(u),
$$

and

$$
\begin{aligned}
U_{n k}(\boldsymbol{\alpha}) & =\partial \mathcal{L}_{n}(\boldsymbol{\alpha}) / \partial \alpha_{k} \\
& =n^{-1} \sum_{i=1}^{n} \int_{0}^{\tau} I\left(C_{i} \geq u\right) K_{h}(u-t)((u-t) / h)^{k}\left\{z_{i}-S_{n, 1}(u, \boldsymbol{\alpha}) / S_{n, 0}(u, \boldsymbol{\alpha})\right\} d \widetilde{N}_{i}(u) .
\end{aligned}
$$

By the assumption of Condition 3, we have $w(h)=\sup _{\left|t-t^{\prime}\right| \leq h}\left|\alpha_{k}(t)-\alpha_{k}\left(t^{\prime}\right)\right|=O(h)$. In this, we consider $\alpha_{k}$ in the neighborhood of zero, that is $\alpha_{k} \in \mathcal{N}_{0}$. And we take $\epsilon=\epsilon_{k}=$ $\max \left\{2 w(h), 6 l_{n} /\left(\mu_{2 k} M_{1}\right)\right\}$. Now, we consider $\alpha_{k} \in\left(-\epsilon_{k}, \epsilon_{k}\right)$, without loss of generality, we assume $\epsilon_{k}<1$. Define

$$
U_{n k}\left(\epsilon_{k}\right)=n^{-1} \sum_{i=1}^{n} \int_{0}^{\tau} I\left(C_{i} \geq 0\right) K_{h}(u-t)((u-t) / h)^{k}\left(z_{i}-S_{n, 1}\left(\epsilon_{k}, u\right) / S_{n, 0}\left(\epsilon_{k}, u\right)\right) d \widetilde{N}_{i}(u),
$$

with

$$
S_{n, j}\left(\epsilon_{k}, u\right)=\sum_{i=1}^{n} I\left(C_{i} \geq 0\right) \exp \left(\epsilon_{k} z_{i}((u-t) / h)^{k}+\boldsymbol{\beta}^{* \mathrm{~T}} z_{i}(\boldsymbol{u})\right) o_{i}(u) z_{i}^{j}, \quad j=0,1,2 .
$$

So by Lemma B1 and Lemma B2, we have (as $n \rightarrow \infty$ ) a.s., for any $t \in T$,

$$
\left|U_{n k}\left( \pm \epsilon_{k}\right)-E\left(U_{n k}\left( \pm \epsilon_{k}\right)\right)\right| \leq l_{n}
$$

where $l_{n}=O\left((\log n /(n h))^{1 / 2}\right)$.

Under conditions 1-5, and by Lemma A1, we have,

$$
E\left(U_{n k}\left(\epsilon_{k}\right)\right)=\int_{0}^{\tau} K_{h}(u-t)((u-t) / h)^{k}\left\{q_{1}(u)-q_{0}(u) S_{1}\left(\epsilon_{k}, u\right) / S_{0}\left(\epsilon_{k}, u\right)\right\} d u,
$$

where

$$
\begin{aligned}
& q_{j}(u)=E\left(p_{1}(u \mid z) p_{2}(u \mid z) \mu_{0}(u) \exp (\beta(u) z) z^{j}\right), \quad j=0,1,2 . \\
& S_{j}\left(\epsilon_{k}, u\right)=E\left(p_{1}(u \mid z) p_{2}(u \mid z) \exp \left(\epsilon_{k} z((u-t) / h)^{k}+\boldsymbol{\beta}^{* \mathrm{~T}} z(\boldsymbol{u})\right) z^{j}\right), \quad j=0,1,2 .
\end{aligned}
$$

Let $(u-t) / h=v$, and $h$ sufficiently small, by Taylor expansion, we have,

$$
\begin{aligned}
& E\left(U_{n k}\left(\epsilon_{k}, u\right)\right)=\int K(v) v^{k}\left\{q_{1}(t)-q_{0}(t) E\left(p_{1}(t \mid z) p_{2}(t \mid z) z \exp \left(\epsilon_{k} z v^{k}+\beta(t) z\right)\right) / E\left(p_{1}(t \mid z)\right.\right. \\
& \left.\left.p_{2}(t \mid z) \exp \left(\epsilon_{k} z v^{k}+\beta(t) z\right)\right)\right\} d v+O(h) .
\end{aligned}
$$


For

$$
\exp \left(\epsilon_{k} z v^{k}+\beta(t) z\right)=\exp (\beta(t) z) \exp \left(\epsilon_{k} v^{k} z\right)=\exp (\beta(t) z)\left(1+\epsilon_{k} v^{k} z+o\left(\epsilon_{k}\right)\right) .
$$

Then

$$
\begin{aligned}
E\left(U_{n k}\left(\epsilon_{k}\right)\right) & =\int K(v) v^{k}\left\{q_{1}(t)-q_{0}(t)\left(q_{1}(t)+q_{2}(t) \epsilon_{k} v^{k}\right) /\left(q_{0}(t)+q_{1}(t) \epsilon_{k} v^{k}\right)\right\} d v+o\left(\epsilon_{k}\right) . \\
& =-\int K(v) v^{2 k} \sigma_{1}(t) \epsilon_{k} /\left(1+o\left(\epsilon_{k}\right)+\epsilon_{k} v^{k} q_{1}(t) / q_{0}(t)\right) d v
\end{aligned}
$$

Similarly,

$$
E\left(U_{n k}\left(-\epsilon_{k}\right)\right)=\int K(v) v^{2 k} \sigma_{1}(t) \epsilon_{k} /\left(1+o\left(\epsilon_{k}\right)-\epsilon_{k} v^{k} q_{1}(t) / q_{0}(t)\right) d v .
$$

Hence, under Condition 5, we have,

$$
E\left(U_{n k}\left(\epsilon_{k}\right)\right) \leq-3^{-1} \mu_{2 k} M_{1} \epsilon_{k},
$$

and

$$
E\left(U_{n k}\left(-\epsilon_{k}\right)\right) \geq 3^{-1} \mu_{2 k} M_{1} \epsilon_{k} .
$$

Therefore, combing (B27), (B29) and (B30), we obtain that (as $n \rightarrow \infty)$ a.s., for any $t \in T$,

$$
U_{n k}\left(\epsilon_{k}\right) \leq l_{n}-3^{-1} \mu_{2 k} M_{1} \epsilon_{k}<0
$$

and

$$
U_{n k}\left(-\epsilon_{k}\right) \geq-l_{n}+3^{-1} \mu_{2 k} M_{1} \epsilon_{k}>0 .
$$

Then the two above inequalities imply that a.s., for any $t \in T$, there exists $\widehat{\alpha}_{k}(t)=\widehat{\alpha}_{k} \in\left(-\epsilon_{k}, \epsilon_{k}\right)$, such that $U_{n k}\left(\widehat{\alpha}_{k}(t)\right)=0$, and $\widehat{\alpha}_{k}(t)=h^{k}\left(\widehat{\beta}_{k}(t)-\beta^{(k)}(t) / k !\right)$. Thus, we have,

$$
\sup _{t \in T}\left|\widehat{\alpha}_{k}(t)\right| \leq \epsilon_{k}, \quad \text { a.s. }
$$

and the above proof follows from $\epsilon_{k}=O\left((\log n /(n h))^{1 / 2}+h\right)$. Hence,

$$
\left.\sup _{t \in T}\left|\widehat{\beta}_{k}(t)-\beta^{(k)}(t) / k !\right|=O\left(h^{-k}\{\log n /(n h))^{1 / 2}+h\right\}\right), \quad \text { a.s. }
$$

then (13) holds.

Proof of Theorem 2. The proof of the asymptotic normality for the coefficient estimator is basically based on the functional central limit theorem of Pollard (1990). Similar to the proof of the Theorem 2.1 of Bilias et al. (1997), we will first show the asymptotic distribution of stochastic functions by the following lemma, which play a crucial role in the proof of Theorem 2 .

LEMMA B3. For any nonzero vector $\boldsymbol{a}=\left(a_{1}, \ldots, a_{p}\right)^{\mathrm{T}}$, let

$$
\begin{aligned}
& u_{1}(s)=n^{-1}(n h)^{1 / 2} \sum_{i=1}^{n} \int_{0}^{s} K_{h}(u-t) \boldsymbol{a}^{\mathrm{T}}(\boldsymbol{u}-\boldsymbol{t}) d M_{i}(u), \\
& u_{2}(s)=n^{-1}(n h)^{1 / 2} \sum_{i=1}^{n} \int_{0}^{s} K_{h}(u-t) \boldsymbol{a}^{\mathrm{T}}(\boldsymbol{u}-\boldsymbol{t}) z_{i} d M_{i}(u),
\end{aligned}
$$

where

$$
d M_{i}(u)=I\left(C_{i} \geq u\right)\left\{d \tilde{N}_{i}(u)-\mu_{0}(u) \exp \left(\beta(u) z_{i}\right) d O_{i}(u)\right\} .
$$


Under Conditions 1-5, we have $\left\{u_{1}(s), s \in T\right\}$ and $\left\{u_{2}(s), s \in T\right\}$ converges in distribution to Gaussian processes $\xi_{1}$ and $\xi_{2}$, respectively, with continuous sample paths, mean 0 and covariance functions identified by

$$
\begin{aligned}
& E\left(\xi_{1}\left(s_{1}\right) \xi_{1}^{\prime}\left(s_{2}\right)\right)=\int_{0}^{s_{1} \wedge s_{2}} h K_{h}^{2}(u-t)\left(\boldsymbol{a}^{\mathrm{T}}(\boldsymbol{u}-\boldsymbol{t})\right)^{2} E\left(p_{1}(u \mid z) p_{2}(u \mid z) \sigma(u \mid z)\right) d u, \\
& E\left(\xi_{2}\left(s_{1}\right) \xi_{2}^{\prime}\left(s_{2}\right)\right)=\int_{0}^{s_{1} \wedge s_{2}} h K_{h}^{2}(u-t)\left(\boldsymbol{a}^{\mathrm{T}}(\boldsymbol{u}-\boldsymbol{t})\right)^{2} E\left(p_{1}(u \mid z) p_{2}(u \mid z) z^{2} \sigma(u \mid z)\right) d u .
\end{aligned}
$$

Proof. Since $u_{1}$ is a special case of $u_{2}$, when we use 1 substitute for $z_{i}$ in (B31), we only need to prove the convergence for $u_{2}$. In order to get the desired convergence, Theorem 10.7 (the functional central limit theorem) of Pollard (1990) was invoked. Therefore conditions (i)-(v) need to be verified.

To verify (i), using the lemma A.1 of Bilias et al. (1997), it suffices to show both $\left\{\int_{0}^{s} K_{h}(u-t) \boldsymbol{a}^{\mathrm{T}}(\boldsymbol{u}-\right.$ $\left.\boldsymbol{t}) I\left(C_{i} \geq u\right) z_{i} d \widetilde{N}_{i}(u), s \in T\right\}$ and $\left\{\int_{0}^{s} K_{h}(u-t) \boldsymbol{a}^{\mathrm{T}}(\boldsymbol{u}-\boldsymbol{t}) I\left(C_{i} \geq u\right) \mu_{0}(u) \exp \left(\beta(u) z_{i}\right) d O_{i}(u), s \in\right.$ $T\}$ are manageable. Without loss of generality, we assume $\boldsymbol{a}^{\mathrm{T}}(\boldsymbol{u}-\boldsymbol{t})>0$ and $z_{i}>0$. Thus, for each $i, \int_{0}^{s} K_{h}(u-t) \boldsymbol{a}^{\mathrm{T}}(\boldsymbol{u}-\boldsymbol{t}) I\left(C_{i} \geq u\right) z_{i} d \widetilde{N}_{i}(u)$ is nondecreasing in $s$. Then it has pseudodimension at most 1. By Theorem 4.8 of Pollard (1990), therefore it must be Euclidean and manageable. Similarly, $\left\{\int_{0}^{s} K_{h}(u-t) \boldsymbol{a}^{\mathrm{T}}(\boldsymbol{u}-\boldsymbol{t}) I\left(C_{i} \geq u\right) \mu_{0}(u) \exp \left(\beta(u) z_{i}\right) d O_{i}(u), s \in T\right\}$ are also Euclidean and manageable. Thus (i) holds.

To verify (ii), under Conditions 1-5 and lemma A1,

$$
\begin{aligned}
& \lim _{n \rightarrow \infty} E\left(u_{2}\left(s_{1}\right) u_{2}\left(s_{2}\right)\right) \\
& =\lim _{n \rightarrow \infty} n^{-1} h \sum_{i=1}^{n} E\left(\left(\int_{0}^{s_{1}} K_{h}(u-t) \boldsymbol{a}^{\mathrm{T}}(\boldsymbol{u}-\boldsymbol{t}) z_{i} d M_{i}(u)\right)\left(\int_{0}^{s_{2}} K_{h}(u-t) \boldsymbol{a}^{\mathrm{T}}(\boldsymbol{u}-\boldsymbol{t}) z_{i} d M_{i}(u)\right)\right) \\
& =\int_{0}^{s_{1} \wedge s_{2}} h K_{h}^{2}(u-t)\left(\boldsymbol{a}^{\mathrm{T}}(\boldsymbol{u}-\boldsymbol{t})\right)^{2} E\left(p_{1}(u \mid z) p_{2}(u \mid z) z^{2} \sigma(u \mid z)\right) d u .
\end{aligned}
$$

Thus (ii) holds. By the classical multivariate central limit theorem, we obtain that the convergence of finite-dimensional distributions of $u_{2}$ to those of $\xi_{2}$ is straightforward. The latter issue is tightness.

For (iii), (iv), under Conditions 2 and 3 , envelops can be chosen as $B^{*} / \sqrt{ } n$, for some constant $B^{*}$. Thus (iii) and (iv) holds.

To test (v), for any $s_{1}, s_{2} \in T$, define

$$
\rho_{n}\left(s_{1}, s_{2}\right)=E\left(u_{2}\left(s_{1}\right)-u_{2}\left(s_{2}\right)\right)^{2}, \quad \rho\left(s_{1}, s_{2}\right)=E\left(\xi_{2}\left(s_{2}\right)-\xi_{2}\left(s_{1}\right)\right)^{2} .
$$

Here,

$$
\begin{aligned}
& \rho_{n}\left(s_{1}, s_{2}\right)=E\left(u_{2}\left(s_{2}\right)-u_{2}\left(s_{1}\right)\right)^{2} \\
& =n^{-1} \sum_{i=1}^{n} E\left(h\left(\int_{s_{1}}^{s_{2}} K_{h}(u-t) \boldsymbol{a}^{\mathrm{T}}(\boldsymbol{u}-\boldsymbol{t}) z_{i} d M_{i}(u)\right)^{2}\right) \\
& =n^{-1} \sum_{i=1}^{n} E\left(\left|\int_{s_{1}}^{s_{2}} h K_{h}^{2}(u-t)\left(\boldsymbol{a}^{\mathrm{T}}(\boldsymbol{u}-\boldsymbol{t})\right)^{2} z_{i}^{2} I\left(C_{i} \geq u\right) \mu_{0}^{2}(u) \exp \left(2 \beta(u) z_{i}\right) o_{i}(u) d u\right|\right) .
\end{aligned}
$$

Clearly, $\rho_{n}$ is equicontinuous on $T$, and $\lim _{n \rightarrow \infty} \rho_{n}\left(s_{1}, s_{2}\right)=\rho\left(s_{1}, s_{2}\right), \rho$ is pseudometric on $T$. Thus $\rho_{n}$ converges to $\rho$, uniformly on $T$. Furthermore, we set $\left\{s_{1}^{n}\right\},\left\{s_{2}^{n}\right\}$ be any two sequences in $T$, it follows that if $\rho\left(s_{1}^{n}, s_{2}^{n}\right) \rightarrow 0$, then $\rho_{n}\left(s_{1}^{n}, s_{2}^{n}\right) \rightarrow 0$. Thus (v) holds.

Therefore, using Theorem 10.7 (the functional central limit theorem) of Pollard (1990), we can state $u_{2}$ converges in distribution to Gaussian process on $T$ having continuous sample path. Hence, $\left\{u_{1}(s), s \in T\right\}$ and $\left\{u_{2}(s), s \in T\right\}$ converges in distribution to Gaussian processes $\xi_{1}$ and $\xi_{2}$, respectively. 
Now, we prove the Theorem 2. Let $\gamma_{n}=(n h)^{-1 / 2}, \boldsymbol{\alpha}=\gamma_{n}^{-1} H\left(\boldsymbol{\beta}-\boldsymbol{\beta}^{*}\right)$, then

$$
\begin{aligned}
& X_{n}\left(\gamma_{n} \boldsymbol{\alpha}, \tau\right)=n^{-1} \sum_{i=1}^{n} \int_{0}^{\tau} I\left(C_{i} \geq u\right) K_{h}(u-t)\left[\gamma_{n} \boldsymbol{\alpha}^{\mathrm{T}} \tilde{z}_{i}(\boldsymbol{u})-\log \left\{\sum _ { i = 1 } ^ { n } I ( C _ { i } \geq u ) \operatorname { e x p } \left(\gamma_{n} \boldsymbol{\alpha}^{\mathrm{T}} \tilde{z}_{j}(\boldsymbol{u})\right.\right.\right. \\
& \left.\left.\left.+\boldsymbol{\beta}^{* \mathrm{~T}} z_{j}(\boldsymbol{u})\right) o_{j}(u) / \sum_{i=1}^{n} I\left(C_{i} \geq u\right) \exp \left(\boldsymbol{\beta}^{* \mathrm{~T}} z_{j}(\boldsymbol{u})\right) o_{j}(u)\right\}\right] d \widetilde{N}_{i}(u) .
\end{aligned}
$$

Let

$$
I\left(C_{i} \geq u\right) d \widetilde{N}_{i}(u)=d M_{i}(u)+I\left(C_{i} \geq u\right) \mu_{0}(u) \exp \left(\beta(u) z_{i}\right) d O_{i}(u)
$$

then

$$
X_{n}\left(\gamma_{n} \boldsymbol{\alpha}, \tau\right)=A_{n}\left(\gamma_{n} \boldsymbol{\alpha}, \tau\right)+U_{n}\left(\gamma_{n} \boldsymbol{\alpha}, \tau\right),
$$

where

$$
\begin{aligned}
& A_{n}\left(\gamma_{n} \boldsymbol{\alpha}, \tau\right)=n^{-1} \sum_{i=1}^{n} \int_{0}^{\tau} K_{h}(u-t)\left[\gamma_{n} \boldsymbol{\alpha}^{\mathrm{T}} \tilde{z}_{i}(\boldsymbol{u})-\log \left\{S_{n, 0}\left(u, \gamma_{n} \boldsymbol{\alpha}\right) / \widetilde{S}_{n, 0}(u)\right\}\right] I\left(C_{i} \geq u\right) \\
& \mu_{0}(u) \exp \left(\beta(u) z_{i}\right) o_{i}(u) d u \\
& U_{n}\left(\gamma_{n} \boldsymbol{\alpha}, \tau\right)=n^{-1} \sum_{i=0}^{n} \int_{0}^{\tau} K_{h}(u-t)\left[\gamma_{n} \boldsymbol{\alpha}^{\mathrm{T}} \tilde{z}_{i}(\boldsymbol{u})-\log \left\{S_{n, 0}\left(u, \gamma_{n} \boldsymbol{\alpha}\right) / \widetilde{S}_{n, 0}(u)\right\}\right] d M_{i}(u) .
\end{aligned}
$$

For

$$
A_{n}\left(\gamma_{n} \boldsymbol{\alpha}, \tau\right)=\int_{0}^{\tau} K_{h}(u-t)\left[\widetilde{S}_{n, 1}^{*}(u)^{\mathrm{T}} \gamma_{n} \boldsymbol{\alpha}-\log \left\{S_{n, 0}\left(u, \gamma_{n} \boldsymbol{\alpha}\right) / \widetilde{S}_{n, 0}(u)\right\} \widetilde{S}_{n, 0}^{*}(u)\right] \mu_{0}(u) d u,
$$

by Taylor expansion of $S_{n, 0}\left(u, \gamma_{n} \boldsymbol{\alpha}\right)$ at $\boldsymbol{\alpha}=0$, it follows that

$$
\begin{aligned}
& \log \left\{S_{n, 0}\left(u, \gamma_{n} \boldsymbol{\alpha}\right) / \widetilde{S}_{n, 0}(u)\right\} \\
& =\left(\widetilde{S}_{n, 1}(u) / \widetilde{S}_{n, 0}(u)\right)^{\mathrm{T}} \gamma_{n} \boldsymbol{\alpha}+2^{-1} \gamma_{n}^{2} \boldsymbol{\alpha}^{\mathrm{T}}\left[\widetilde{S}_{n, 2}(u) / \widetilde{S}_{n, 0}(u)-\left(\widetilde{S}_{n, 1}(u) / \widetilde{S}_{n, 0}(u)\right)^{\otimes 2}\right] \boldsymbol{\alpha}+o_{p}\left(\gamma_{n}^{2}\right) \\
& =\left(\widetilde{S}_{1}(u) / \widetilde{S}_{0}(u)\right)^{\mathrm{T}} \gamma_{n} \boldsymbol{\alpha}+2^{-1} \gamma_{n}^{2} \boldsymbol{\alpha}^{\mathrm{T}}\left\{\widetilde{S}_{2}(u) / \widetilde{S}_{0}(u)-\left(\widetilde{S}_{1}(u) / \widetilde{S}_{0}(u)\right)^{\otimes 2}\right\} \boldsymbol{\alpha}+o_{p}\left(\gamma_{n}^{2}\right) .
\end{aligned}
$$

Hence

$$
A_{n}\left(\gamma_{n} \boldsymbol{\alpha}, \tau\right)=\gamma_{n} A_{n, 1}(\tau)^{\mathrm{T}} \boldsymbol{\alpha}-2^{-1} \gamma_{n}^{2} \boldsymbol{\alpha}^{\mathrm{T}} F_{n, 1}(\tau) \boldsymbol{\alpha}+o_{p}\left(\gamma_{n}^{2}\right)
$$

where

$$
\begin{aligned}
& A_{n, 1}(\tau)=\int_{0}^{\tau} K_{h}(u-t)\left\{\widetilde{S}_{1}^{*}(u)-\widetilde{S}_{1}(u) \widetilde{S}_{0}^{*}(u) / \widetilde{S}_{0}(u)\right\} \mu_{0}(u) d u, \\
& F_{n, 1}(\tau)=\int_{0}^{\tau} K_{h}(u-t)\left\{\widetilde{S}_{2}(u) / \widetilde{S}_{0}(u)-\left(\widetilde{S}_{1}(u) / \widetilde{S}_{0}(u)\right)^{\otimes 2}\right\} \widetilde{S}_{0}^{*}(u) \mu_{0}(u) d u .
\end{aligned}
$$

For $|u-t|<c h$, let $u=t+h v$, under Conditions 1-5, we have

$$
\begin{aligned}
& F_{n, 1}(\tau)=\int K(v)\left\{\widetilde{S}_{2}(t+h v) / \widetilde{S}_{0}(t+h v)-\left(\widetilde{S}_{1}(t+h v) / \widetilde{S}_{0}(t+h v)\right)^{\otimes 2}\right\} \widetilde{S}_{0}^{*}(t+h v) \mu_{0}(t+h v) d v \\
& =\sigma_{1}(t) \Omega_{1}+o_{p}(1),
\end{aligned}
$$

where $\Omega_{1}=\int K(v) \boldsymbol{v}^{\otimes 2} d v$, and $\boldsymbol{v}=\left(1, v, \ldots, v^{p}\right)^{\mathrm{T}}$.

Thus

$$
A_{n}\left(\gamma_{n} \boldsymbol{\alpha}, \tau\right)=\gamma_{n} A_{n, 1}(\tau)^{\mathrm{T}} \boldsymbol{\alpha}-2^{-1} \gamma_{n}^{2} \boldsymbol{\alpha}^{\mathrm{T}} \sigma_{1}(t) \Omega_{1} \boldsymbol{\alpha}+o_{p}\left(\gamma_{n}^{2}\right)
$$


Similarly, we have

$$
U_{n}\left(\gamma_{n} \boldsymbol{\alpha}, \tau\right)=\gamma_{n} \boldsymbol{\alpha}^{\mathrm{T}} U_{n, 1}(\tau)-2^{-1} \gamma_{n}^{2} \boldsymbol{\alpha}^{\mathrm{T}} F_{n, 2}(\tau) \boldsymbol{\alpha}+o_{p}\left(\gamma_{n}^{2}\right),
$$

where

$$
\begin{aligned}
& U_{n, 1}(\tau)=\int_{0}^{\tau} K_{h}(u-t) n^{-1} \sum_{i=1}^{n}\left\{\tilde{z}_{i}(\boldsymbol{u})-\widetilde{S}_{n, 1}(u) / \widetilde{S}_{n, 0}(u)\right\} d M_{i}(u), \\
& F_{n, 2}(\tau)=n^{-1} \sum_{i=1}^{n} \int_{0}^{\tau} K_{h}(u-t)\left\{\widetilde{S}_{n, 2}(u) / \widetilde{S}_{n, 0}(u)-\left(\widetilde{S}_{n, 1}(u) / \widetilde{S}_{n, 0}(u)\right)^{\otimes 2}\right\} d M_{i}(u) .
\end{aligned}
$$

For $F_{n, 2}(\tau)$, similar to Lemma B3, we have $\left\{\int_{0}^{s} K_{h}(u-t) d M_{i}(u), s \in T\right\}$ is manageable. Let constant $\bar{B} / \sqrt{ } n$ as envelope. Thus, using Theorem 8.3 (the uniform law of large numbers) of Pollard (1990), we can derive

$$
\lim _{n \rightarrow \infty} \sup _{s \in T}\left\|n^{-1} \sum_{i=1}^{n} \int_{0}^{s} K_{h}(u-t) d M_{i}(u)-0\right\|=0 .
$$

Also, by Lemma A1, as $n \rightarrow \infty$,

$$
\sup _{s \in T}\left\|\left\{\widetilde{S}_{n, 2}(u) / \widetilde{S}_{n, 0}(u)-\left(\widetilde{S}_{n, 1}(u) / \widetilde{S}_{n, 0}(u)\right)^{\otimes 2}\right\}-\left\{\widetilde{S}_{2}(u) / \widetilde{S}_{0}(u)-\left(\widetilde{S}_{1}(u) / \widetilde{S}_{0}(u)\right)^{\otimes 2}\right\}\right\| \rightarrow 0 .
$$

Then, by lemma A2, we have

$$
F_{n, 2}(\tau)=O_{p}\left(\gamma_{n}\right)
$$

Therefore,

$$
U_{n}\left(\gamma_{n} \boldsymbol{\alpha}, \tau\right)=\gamma_{n} \boldsymbol{\alpha}^{\mathrm{T}} U_{n, 1}(\tau)+O_{p}\left(\gamma_{n}^{2}\right) .
$$

From (B37), (B38) and (B39), we obtain

$$
X_{n}\left(\gamma_{n} \boldsymbol{\alpha}, \tau\right)=\left\{A_{n, 1}(\tau)+U_{n, 1}(\tau)\right\}^{\mathrm{T}} \gamma_{n} \boldsymbol{\alpha}-2^{-1} \gamma_{n}^{2} \boldsymbol{\alpha}^{\mathrm{T}} \sigma_{1}(t) \Omega_{1} \boldsymbol{\alpha}+o_{p}\left(\gamma_{n}^{2}\right) .
$$

Using Quadratic Approximation Lemma of Fan \& Gijbels (1996), we derive

$$
\widehat{\boldsymbol{\alpha}}=\gamma_{n}^{-1}\left(\sigma_{1}(t) \Omega_{1}\right)^{-1}\left\{A_{n, 1}(\tau)+U_{n, 1}(\tau)\right\}+o_{p}(1) .
$$

For $A_{n, 1}(\tau)=\int_{0}^{\tau} K_{h}(u-t)\left\{\widetilde{S}_{1}^{*}(u)-\widetilde{S}_{1}(u) \widetilde{S}_{0}^{*}(u) / \widetilde{S}_{0}(u)\right\} \mu_{0}(u) d u$. We apply Taylor expansion to the term:

$$
\widetilde{S}_{1}^{*}(u)-\widetilde{S}_{1}(u) \widetilde{S}_{0}^{*}(u) / \widetilde{S}_{0}(u)=\widetilde{S}_{1}^{*}(u)-\widetilde{S}_{1}(u)-\widetilde{S}_{1}(u)\left(\widetilde{S}_{0}^{*}(u)-\widetilde{S}_{0}(u)\right) / \widetilde{S}_{0}(u) .
$$

Note that

$$
\begin{aligned}
\beta(u) z & \approx \beta(t) z+\beta^{\prime}(t) z(u-t)+\cdots+\beta^{(p)}(t) z(u-t)^{p} / p !+\beta^{(p+1)}(t) z(u-t)^{p+1} /(p+1) ! \\
& =\boldsymbol{\beta}^{* \mathrm{~T}} z(\boldsymbol{u})+\beta^{(p+1)}(t) z(u-t)^{p+1} /(p+1) ! .
\end{aligned}
$$

Then

$$
\begin{aligned}
\exp (\beta(u) z)-\exp \left(\boldsymbol{\beta}^{* \mathrm{~T}} z(\boldsymbol{u})\right) & \approx \exp (\beta(u) z)\left\{1-\exp \left(-\beta^{(p+1)}(t) z(u-t)^{p+1} /(p+1) !\right)\right\} \\
& \approx \exp (\beta(u) z) \beta^{(p+1)}(t) z(u-t)^{p+1} /(p+1) ! .
\end{aligned}
$$


Thus

$$
\begin{aligned}
& \widetilde{S}_{1}^{*}(u)-\widetilde{S}_{1}(u)=E\left(p_{1}(u \mid z) p_{2}(u \mid z) \exp (\beta(u) z) z \tilde{z}(\boldsymbol{u})\right) \beta^{(p+1)}(t)(u-t)^{p+1} /(p+1) ! \\
& +o\left((u-t)^{p+1}\right) \text {, } \\
& \widetilde{S}_{0}^{*}(u)-\widetilde{S}_{0}(u)=E\left(p_{1}(u \mid z) p_{2}(u \mid z) \exp (\beta(u) z) z\right) \beta^{(p+1)}(t)(u-t)^{p+1} /(p+1) !+o\left((u-t)^{p+1}\right), \\
& \widetilde{S}_{0}(u)=E\left(p_{1}(u \mid z) p_{2}(u \mid z) \exp (\beta(u) z)\right)+O\left((u-t)^{p+1}\right) \text {, } \\
& \widetilde{S}_{1}(u)=E\left(p_{1}(u \mid z) p_{2}(u \mid z) \exp (\beta(u) z) \tilde{z}(\boldsymbol{u})\right)+O\left((u-t)^{p+1}\right) .
\end{aligned}
$$

Therefore, we have

$$
\begin{aligned}
& A_{n, 1}(\tau)=\int_{0}^{\tau} K_{h}(u-t)\left[\left\{E\left(p_{1}(t \mid z) p_{2}(t \mid z) \exp (\beta(u) z) z \tilde{z}(\boldsymbol{u})\right)-\widetilde{S}_{1}^{*}(u) S_{1}^{*}(u, \beta(u)) / S_{0}^{*}(u, \beta(u))\right\}\right. \\
& \left.\beta^{(p+1)}(t)(u-t)^{p+1} /(p+1) !+o\left((u-t)^{p+1}\right)\right] d u .
\end{aligned}
$$

Let $u=t+h v$, we derive

$$
A_{n, 1}(\tau)=\int K(v) \boldsymbol{v} v^{p+1} d v \sigma_{1}(t) h^{p+1} \beta^{(p+1)}(t) /(p+1) !+o\left(h^{p+1}\right) .
$$

From (B40) and (B41), we obtain (let $\left.\boldsymbol{b}=\int K(v) v^{p+1} \boldsymbol{v} d v\right)$

$$
\widehat{\boldsymbol{\alpha}}=\gamma_{n}^{-1} \Omega_{1}^{-1} \boldsymbol{b} h^{p+1} \beta^{(p+1)}(t) /(p+1) !+\gamma_{n}^{-1} \sigma_{1}^{-1}(t) \Omega_{1}^{-1} U_{n, 1}(\tau)+o_{p}(1) .
$$

Hence,

$$
(n h)^{1 / 2}\left\{H\left(\widehat{\boldsymbol{\beta}}-\boldsymbol{\beta}^{*}\right)-\Omega_{1}^{-1} \boldsymbol{b} h^{p+1} \beta^{(p+1)}(t) /(p+1) !\right\}=\gamma_{n}^{-1} \sigma_{1}^{-1}(t) \Omega_{1}^{-1} U_{n, 1}(\tau)+o_{p}(1) .
$$

Therefore, (B40) can be reduced to prove the multivariate normality of $(n h)^{1 / 2} U_{n, 1}(\tau)$. That is equivalent to prove the normality of $\boldsymbol{a}^{\mathrm{T}}(n h)^{1 / 2} U_{n, 1}(\tau)$, for any nonzero vector $\boldsymbol{a}=\left(a_{1}, \ldots, a_{p}\right)^{\mathrm{T}}$. Write $\widetilde{U}_{n}(s)=$ $\boldsymbol{a}^{\mathrm{T}}(n h)^{1 / 2} U_{n, 1}(s)$ is empirical process, we will show that it converges to Gaussian process $\tilde{\xi}$. In fact,

$$
\widetilde{U}_{n}(s)=\widetilde{U}_{n 1}(s)+\widetilde{U}_{n 2}(s),
$$

where

$$
\begin{aligned}
\widetilde{U}_{n 1}(s)= & n^{-1}(n h)^{1 / 2} \sum_{i=1}^{n} \int_{0}^{s} K_{h}(u-t) \boldsymbol{a}^{\mathrm{T}}(\boldsymbol{u}-\boldsymbol{t})\left\{z_{i}-S_{1}\left(u, \boldsymbol{\beta}^{*}\right) / S_{0}\left(u, \boldsymbol{\beta}^{*}\right)\right\} d M_{i}(u), \\
\widetilde{U}_{n 2}(s)= & n^{-1}(n h)^{1 / 2} \sum_{i=1}^{n} \int_{0}^{s} K_{h}(u-t) \boldsymbol{a}^{\mathrm{T}}(\boldsymbol{u}-\boldsymbol{t})\left\{S_{1}\left(u, \boldsymbol{\beta}^{*}\right) / S_{0}\left(u, \boldsymbol{\beta}^{*}\right)-S_{n, 1}\left(u, \boldsymbol{\beta}^{*}\right) / S_{n, 0}\left(u, \boldsymbol{\beta}^{*}\right)\right\} \\
& d M_{i}(u) .
\end{aligned}
$$

For $\widetilde{U}_{n 2}(s)$, by Lemma B3 and the Strong Representation Theorem of Pollard (1990), we can construct a new probability space, and have

$$
\sup _{s \in T}\left\|u_{1}(s)-\xi_{1}(s)\right\| \rightarrow 0, \quad \text { as } \quad n \rightarrow \infty,
$$

and by Lemma A1, we have

$$
\sup _{s \in T}\left\|S_{1}\left(u, \boldsymbol{\beta}^{*}\right) / S_{0}\left(u, \boldsymbol{\beta}^{*}\right)-S_{n, 1}\left(u, \boldsymbol{\beta}^{*}\right) / S_{n, 0}\left(u, \boldsymbol{\beta}^{*}\right)\right\| \rightarrow 0, \quad \text { as } \quad n \rightarrow \infty .
$$


Then by Lemma A2, we can show that almost surely,

$$
\begin{aligned}
& n^{-1}(n h)^{1 / 2} \sum_{i=1}^{n} \int_{0}^{s} K_{h}(u-t) \boldsymbol{a}^{\mathrm{T}}(\boldsymbol{u}-\boldsymbol{t})\left\{S_{1}\left(u, \boldsymbol{\beta}^{*}\right) / S_{0}\left(u, \boldsymbol{\beta}^{*}\right)-S_{n, 1}\left(u, \boldsymbol{\beta}^{*}\right) / S_{n, 0}\left(u, \boldsymbol{\beta}^{*}\right)\right\} d M_{i}(u) \\
& \rightarrow 0, \quad \text { as } \quad n \rightarrow \infty
\end{aligned}
$$

which holds in original probability space since the statement is now in probability. Thus the convergence of $\widetilde{U}_{n}(s)$ reduces to that of $\widetilde{U}_{n 1}(s)$. Here,

$$
\begin{aligned}
\lim _{n \rightarrow \infty} E\left(\widetilde{U}_{n 1}\left(s_{1}\right) \widetilde{U}_{n 1}\left(s_{2}\right)\right) \\
=\lim _{n \rightarrow \infty} n^{-1} \sum_{i=1}^{n} E\left(h ( \int _ { 0 } ^ { s _ { 1 } } K _ { h } ( u - t ) \boldsymbol { a } ^ { \mathrm { T } } ( \boldsymbol { u } - \boldsymbol { t } ) \{ z _ { i } - S _ { 1 } ( u , \boldsymbol { \beta } ^ { * } ) / S _ { 0 } ( u , \boldsymbol { \beta } ^ { * } ) \} d M _ { i } ( u ) ) \left(\int_{0}^{s_{2}} K_{h}(u-t)\right.\right. \\
\left.\left.\boldsymbol{a}^{\mathrm{T}}(\boldsymbol{u}-\boldsymbol{t})\left\{z_{i}-S_{1}\left(u, \boldsymbol{\beta}^{*}\right) / S_{0}\left(u, \boldsymbol{\beta}^{*}\right)\right\} d M_{i}(u)\right)\right) \\
=\int_{0}^{s_{1} \wedge s_{2}} h K_{h}^{2}(u-t)\left(\boldsymbol{a}^{\mathrm{T}}(\boldsymbol{u}-\boldsymbol{t})\right)^{2} E\left(p_{1}(u \mid z) p_{2}(u \mid z)\left\{z-S_{1}\left(u, \boldsymbol{\beta}^{*}\right) / S_{0}\left(u, \boldsymbol{\beta}^{*}\right)\right\}^{2} \mu_{0}^{2}(u) \exp (2\right. \\
\beta(u) z)) d u \\
=E\left(\tilde{\xi}\left(s_{1}\right) \tilde{\xi}\left(s_{2}\right)\right) .
\end{aligned}
$$

Then, the convergence of finite-dimensional distributions of $\widetilde{U}_{n 1}(s)$ to those of $\tilde{\xi}$ is clearly true by the classical multivariate central limit theorem, since $\widetilde{U}_{n 1}$ is a sum of independent random variables. It remains to show tightness for $\widetilde{U}_{n 1}$, or equivalently, tightness for

$$
\widetilde{U}_{n 1}(s)=n^{-1}(n h)^{1 / 2} \sum_{i=1}^{n} \int_{0}^{s} K_{h}(u-t) \boldsymbol{a}^{\mathrm{T}}(\boldsymbol{u}-\boldsymbol{t})\left\{z_{i}-S_{1}\left(u, \boldsymbol{\beta}^{*}\right) / S_{0}\left(u, \boldsymbol{\beta}^{*}\right)\right\} d M_{i}(u) .
$$

By Lemma B3, $\left\{n^{-1}(n h)^{1 / 2} \sum_{i=1}^{n} \int_{0}^{s} K_{h}(u-t) \boldsymbol{a}^{\mathrm{T}}(\boldsymbol{u}-\boldsymbol{t}) z_{i} d M_{i}(u), s \in T\right\}$ is tightness. And analogous to the proof of Lemma B3, we can check that $\left\{n^{-1}(n h)^{1 / 2} \sum_{i=1}^{n} \int_{0}^{s} K_{h}(u-t) \boldsymbol{a}^{\mathrm{T}}(\boldsymbol{u}-\right.$ $\left.\boldsymbol{t}) S_{1}\left(u, \boldsymbol{\beta}^{*}\right) / S_{0}\left(u, \boldsymbol{\beta}^{*}\right) d M_{i}(u), s \in T\right\}$ is tightness, too. Therefore, $\widetilde{U}_{n 1}(s)$ converges to $\tilde{\xi}$. Hence, $\boldsymbol{a}^{\mathrm{T}}(n h)^{1 / 2} U_{n, 1}(\tau)$ is normal. Then, $(n h)^{1 / 2} U_{n, 1}(\tau)$ is multivariate normal, and asymptotically covariance is as follows:

$$
\Sigma_{2}(t)=\int K^{2}(v) \boldsymbol{v}^{\otimes 2} d v E\left(p_{1}(t \mid z) p_{2}(t \mid z) \mu_{0}^{2}(t) \exp (2 \beta(t) z)\left(z-q_{1}(t) / q_{0}(t)\right)^{2}\right)=\sigma_{2}(t) \Omega_{2},
$$

where $\Omega_{2}=\int K^{2}(v) \boldsymbol{v}^{\otimes 2} d v$.

Therefore,

$$
(n h)^{1 / 2}\left\{H\left(\widehat{\boldsymbol{\beta}}-\boldsymbol{\beta}^{*}\right)-\Omega_{1}^{-1} \boldsymbol{b} h^{p+1} \beta^{(p+1)}(t) /(p+1) !\right\} \rightarrow N\left(0, \sigma_{1}^{-2}(t) \sigma_{2}(t) \Omega_{1}^{-1} \Omega_{2} \Omega_{1}^{-1}\right),
$$

as $n \rightarrow \infty, h \rightarrow 0, n h \rightarrow \infty$.

Proof of consistency of covariance. For $\widehat{\Sigma}(t)=\widehat{\Sigma}_{1}^{-1}(t) \widehat{\Sigma}_{2}(t) \widehat{\Sigma}_{1}^{-1}(t)$, where $\widehat{\Sigma}_{1}(t)$ and $\widehat{\Sigma}_{2}(t)$ defined as (20) and (21), respectively. Next up, we will show that $\widehat{\Sigma}_{1}(t)$ and $\widehat{\Sigma}_{2}(t)$ are consistent, respectively.

First of all, we give a conclusion by the following demonstration. Under Conditions 2-4, there exists a neighborhood $\mathcal{B}$ of $\boldsymbol{\beta}^{*}$, such that functions $S_{j}(u, \boldsymbol{\beta}), j=0,1,2$ are continuous in $\boldsymbol{\beta} \in \mathcal{B}$, uniformly in $u \in T$. And $S_{0}(u, \boldsymbol{\beta})$ is bounded away of from zero on $(u, \boldsymbol{\beta}) \in T \times \mathcal{B}$. Furthermore, by Lemma A1, we can derive, for each $j=0,1,2$,

$$
\sup _{\mathcal{B} \times T}\left\|S_{n, j}(u, \boldsymbol{\beta})-S_{j}(u, \boldsymbol{\beta})\right\| \rightarrow 0, \quad \text { as } \quad n \rightarrow \infty .
$$

Further, account for $\widehat{\Sigma}_{1}(t)$. We will prove $\widehat{\Sigma}_{1}(t)$ converges to $\Sigma_{1}(t)=\sigma_{1}(t) \Omega_{1}$. Let

$$
v_{1}(u, \beta(u))=S_{2}^{*}(u, \beta(u)) / S_{0}^{*}(u, \beta(u))-S_{1}^{* 2}(u, \beta(u)) / S_{0}^{* 2}(u, \beta(u)),
$$


and from the defined $q_{j}(t)$, we have $S_{j}^{*}(t, \beta(t))=q_{j}(t) / \mu_{0}(t), j=0,1,2$.

Then, we obtain

$$
\begin{aligned}
\Sigma_{1}(t) & =\left\{q_{2}(t)-q_{1}^{2}(t) / q_{0}(t)\right\} \Omega_{1}=\mu_{0}(t)\left\{S_{2}^{*}(t, \beta(t))-S_{1}^{* 2}(t, \beta(t)) / S_{0}^{*}(t, \beta(t))\right\} \Omega_{1} \\
& =\int K_{h}(u-t)(\boldsymbol{u}-\boldsymbol{t})^{\otimes 2} \mu_{0}(u) S_{0}^{*}(u, \beta(u)) v_{1}(u, \beta(u)) d u+o(1) .
\end{aligned}
$$

Using triangle inequality, we have

$$
\begin{aligned}
& \left\|\widehat{\Sigma}_{1}(t)-\Sigma_{1}(t)\right\| \\
& \leq\left\|n^{-1} \sum_{i=1}^{n} \int_{0}^{\tau} I\left(C_{i} \geq u\right) K_{h}(u-t)(\boldsymbol{u}-\boldsymbol{t})^{\otimes 2}\left(V_{1}(u, \widehat{\boldsymbol{\beta}})-v_{1}(u, \beta(u))\right) d \widetilde{N}_{i}(u)\right\| \\
& +\left\|n^{-1} \sum_{i=1}^{n} \int_{0}^{\tau} I\left(C_{i} \geq u\right) K_{h}(u-t)(\boldsymbol{u}-\boldsymbol{t})^{\otimes 2} v_{1}(u, \beta(u))\left\{d \widetilde{N}_{i}(u)-\mu_{0}(u) \exp \left(\beta(u) z_{i}\right) o_{i}(u) d u\right\}\right\| \\
& +\| n^{-1} \sum_{i=1}^{n} \int_{0}^{\tau} I\left(C_{i} \geq u\right) K_{h}(u-t)(\boldsymbol{u}-\boldsymbol{t})^{\otimes 2} v_{1}(u, \beta(u)) \mu_{0}(u) \exp \left(\beta(u) z_{i}\right) o_{i}(u) d u-\int_{0}^{\tau} K_{h}(u \\
& -t)(\boldsymbol{u}-\boldsymbol{t})^{\otimes 2} \mu_{0}(u) S_{0}^{*}(u, \beta(u)) v_{1}(u, \beta(u)) d u \| \\
& +\| \int_{0}^{\tau} K_{h}(u-t)(\boldsymbol{u}-\boldsymbol{t})^{\otimes 2} \mu_{0}(u) S_{0}^{*}(u, \beta(u)) v_{1}(u, \beta(u)) d u-\mu_{0}(t)\left\{S_{2}^{*}(t, \beta(t))-S_{1}^{* 2}(t, \beta(t)) /\right. \\
& \left.S_{0}^{*}(t, \beta(t))\right\} \Omega_{1} \| .
\end{aligned}
$$

where $V_{1}(u, \widehat{\boldsymbol{\beta}})$ defined in (22).

For the first term of the right-hand side, under Conditions 1-5, by the consequence of Theorem 1, from (5), we can derive

$$
\sup _{\mathcal{B} \times T}\left\|S_{j}(u, \widehat{\boldsymbol{\beta}})-S_{j}^{*}(u, \beta(u))\right\| \rightarrow 0, \quad \text { as } \quad n \rightarrow \infty .
$$

Hence, from (B43) and (B46), we have

$$
\sup _{\mathcal{B} \times T}\left\|V_{1}(u, \widehat{\boldsymbol{\beta}})-v_{1}(u, \beta(u))\right\| \rightarrow 0, \quad \text { as } n \rightarrow \infty .
$$

By consequence of Lenglart inequality,

$$
\begin{aligned}
& \operatorname{pr}\left(\left\{n^{-1} \sum_{i=1}^{n} \int_{0}^{\tau} I\left(C_{i} \geq u\right) K_{h}(u-t) d \widetilde{N}_{i}(u)>C\right\}\right) \\
& \leq \frac{\delta}{C}+\operatorname{pr}\left(\left\{\int_{0}^{\tau} n^{-1} \sum_{i=1}^{n} I\left(C_{i} \geq u\right) K_{h}(u-t) \mu_{0}(u) \exp \left(\beta(u) z_{i}\right) o_{i}(u) d u>\delta\right\}\right),
\end{aligned}
$$

when $\delta>\int_{0}^{\tau} K_{h}(u-t) \mu_{0}(u) S_{0}^{*}(u, \beta(u)) d u=\mu_{0}(t) S_{0}^{*}(t, \beta(t))$, the latter probability tends to zero as $n \rightarrow \infty, h \rightarrow 0$, and $n h \rightarrow \infty$. Thus the first term converges to zero.

For the second term of the right-hand side, that is $n^{-1} \sum_{i=1}^{n} \int_{0}^{\tau} K_{h}(u-t) v_{1}(u, \beta(u)) d M_{i}(u)$ is empirical process. by Lemma B3 and $v_{1}(u, \beta(u))$ is non-negative function, analogous to the proof of Theorem 2, using the Theorem 8.3 (the uniform law of large numbers) of Pollard (1990), we can demonstrate the second term converges to zero.

For the third term of the right-hand side, under Conditions 1-4, functions $v_{1}(u, \beta(u))$ are bounded. So from (B43), it is easy to prove the third term tend to zero.

For the fourth term of the right-hand side, from (B45), obviously, it converges to zero.

Therefore,

$$
\left\|\widehat{\Sigma}_{1}(t)-\Sigma_{1}(t)\right\| \rightarrow 0, \quad \text { as } \quad n \rightarrow \infty
$$


Next, we will prove $\widehat{\Sigma}_{2}(t)$ converges to $\Sigma_{2}(t)=\sigma_{2}(t) \Omega_{2}$ by the following demonstration. Let

$$
v_{2}(u, \beta(u))=\left\{z-S_{1}^{*}(u, \beta(u)) / S_{0}^{*}(u, \beta(u))\right\}^{2} .
$$

For

$$
\begin{aligned}
\Sigma_{2}(t) & =E\left(p_{1}(t \mid z) p_{2}(t \mid z) \mu_{0}^{2}(t) \exp (2 \beta(t) z)\left(z-q_{1}(t) / q_{0}(t)\right)^{2}\right) \Omega_{2} \\
& =\int_{0}^{\tau} h K_{h}^{2}(u-t)(\boldsymbol{u}-\boldsymbol{t})^{\otimes 2} \mu_{0}^{2}(u) E\left(p_{1}(u \mid z) p_{2}(u \mid z) \exp (2 \beta(u) z) v_{2}(u, \beta(u)) d u+o(1) .\right.
\end{aligned}
$$

Then, using triangle inequality, we have

$$
\begin{aligned}
& \left\|\widehat{\Sigma}_{2}(t)-\Sigma_{2}(t)\right\| \\
& \leq \| n^{-1} \sum_{i=1}^{n} \int_{0}^{\tau} h K_{h}^{2}(u-t)(\boldsymbol{u}-\boldsymbol{t})^{\otimes 2} I\left(C_{i} \geq u\right) V_{2}(u, \widehat{\boldsymbol{\beta}}) \widehat{\mu}_{0}^{2}(u, \widehat{\beta}(u)) \exp \left(2 \widehat{\beta}(t) z_{i}\right) o_{i}(u) d u-\int_{0}^{\tau} \\
& h K_{h}^{2}(u-t)(\boldsymbol{u}-\boldsymbol{t})^{\otimes 2} \widehat{\mu}_{0}^{2}(u, \widehat{\beta}(u)) E\left(p_{1}(u \mid z) p_{2}(u \mid z) v_{2}(u, \beta(u)) \exp (2 \beta(t) z)\right) d u \| \\
& +\| \int_{0}^{\tau} h K_{h}^{2}(u-t)(\boldsymbol{u}-\boldsymbol{t})^{\otimes 2}\left\{\widehat{\mu}_{0}^{2}(u, \widehat{\beta}(u))-\mu_{0}^{2}(u)\right\} E\left(p_{1}(u \mid z) p_{2}(u \mid z) v_{2}(u, \beta(u)) \exp (2 \beta(t) z)\right) \\
& d u \| \\
& +\| \int_{0}^{\tau} h K_{h}^{2}(u-t)(\boldsymbol{u}-\boldsymbol{t})^{\otimes 2} \mu_{0}^{2}(u) E\left(p_{1}(u \mid z) p_{2}(u \mid z) v_{2}(u, \beta(u)) \exp (2 \beta(t) z)\right) d u-E\left(p_{1}(t \mid z)\right. \\
& \left.p_{2}(t \mid z) \mu_{0}^{2}(t) \exp (2 \beta(t) z)\left(z-q_{1}(t) / q_{0}(t)\right)^{2}\right) \Omega_{2} \| .
\end{aligned}
$$

where $V_{2}(u, \widehat{\boldsymbol{\beta}})$ defined in (23).

For the first term of the right-hand side, let $V_{21}(u, \widehat{\boldsymbol{\beta}})=S_{n, 1}(u, \widehat{\boldsymbol{\beta}}) / S_{n, 0}(u, \widehat{\boldsymbol{\beta}})$, and $v_{21}=$ $S_{1}^{*}(u, \beta(u)) / S_{0}^{*}(u, \beta(u))$. We have

$$
\begin{aligned}
& n^{-1} \sum_{i=1}^{n} \int_{0}^{\tau} h K_{h}^{2}(u-t)(\boldsymbol{u}-\boldsymbol{t})^{\otimes 2} I\left(C_{i} \geq u\right) V_{2}(u, \widehat{\boldsymbol{\beta}}) \widehat{\mu}_{0}^{2}(u, \widehat{\beta}(u)) \exp \left(2 \widehat{\beta}(t) z_{i}\right) o_{i}(u) d u \\
& =\int_{0}^{\tau} h K_{h}^{2}(u-t)(\boldsymbol{u}-\boldsymbol{t})^{\otimes 2} \widehat{\mu}_{0}^{2}(u, \widehat{\beta}(u))\left\{S_{n, 2}^{*}(u, 2 \widehat{\beta}(t))-2 S_{n, 1}^{*}(u, 2 \widehat{\beta}(t)) V_{21}(u, \widehat{\boldsymbol{\beta}})+S_{n, 0}^{*}(u, 2 \widehat{\beta}(t))\right. \\
& \left.V_{21}^{2}(u, \widehat{\boldsymbol{\beta}})\right\} d u .
\end{aligned}
$$

From (14) and (B43), we can derive, for each $j=0,1,2$,

$$
\sup _{\mathcal{B} \times T}\left\|S_{n, j}(u, 2 \widehat{\beta}(t))-S_{j}(u, 2 \beta(t))\right\| \rightarrow 0, \quad \text { as } \quad n \rightarrow \infty .
$$

Analogous to the proof of $V_{1}(u, \widehat{\boldsymbol{\beta}})$, we can obtain

$$
\sup _{\mathcal{B} \times T}\left\|V_{21}(u, \widehat{\boldsymbol{\beta}})-v_{21}(u, \beta(u))\right\| \rightarrow 0, \quad \text { as } \quad n \rightarrow \infty,
$$

and

$$
\sup _{\mathcal{B} \times T}\left\|V_{21}^{2}(u, \widehat{\boldsymbol{\beta}})-v_{21}^{2}(u, \beta(u))\right\| \rightarrow 0, \quad \text { as } \quad n \rightarrow \infty .
$$

Hence, from (B52), (B53), (B54) and (B55), the convergence of the first term can be demonstrated.

For the second term of the right-hand side, let

$$
\widehat{\mu}_{0}^{2}(u, \widehat{\beta}(u))-\mu_{0}^{2}(u)=\left(\widehat{\mu}_{0}(u, \widehat{\beta}(u))+\mu_{0}(u)\right)\left(\widehat{\mu}_{0}(u, \widehat{\beta}(u))-\mu_{0}(u)\right),
$$


and

$$
\begin{aligned}
\widehat{\mu}_{0}(u, \widehat{\beta}(u))-\mu_{0}(u) & =\left\{\widehat{\mu}_{0}(u, \widehat{\beta}(u))-\widehat{\mu}_{0}(u, \beta(u))\right\}+\left\{\widehat{\mu}_{0}(u, \beta(u))-\mu_{0}(u)\right\} \\
& =H_{n}\left(u, \beta^{*}\right)(\widehat{\beta}(u)-\beta(u))+n^{-1} \sum_{i=1}^{n} d M_{i}(u) / S_{n, 0}^{*}(u, \beta(u))
\end{aligned}
$$

where $H_{n}\left(u, \beta^{*}\right)=-S_{n, 1}^{*}\left(u, \beta^{*}\right) \sum_{i=1}^{n} I\left(C_{i} \geq u\right) N_{i}(u) o_{i}(u) / n S_{n, 0}^{* 2}\left(u, \beta^{*}\right)$, and $\beta^{*}$ is between $\beta(u)$ and $\widehat{\beta}(u)$.

Under Conditions $1-5$, by Lemma $\mathrm{A} 1$ and (14), $H_{n}\left(u, \beta^{*}\right)$ is bounded, and $\widehat{\beta}(u)$ uniform converges to $\beta(u)$. Hence, we can derive

$$
\sup _{u \in T}\left\|H_{n}\left(u, \beta^{*}\right)(\widehat{\beta}(u)-\beta(u))\right\| \rightarrow 0, \quad \text { as } \quad n \rightarrow \infty
$$

For $n^{-1} \sum_{i=1}^{n} d M_{i}(u) / S_{n, 0}^{*}(u, \beta(u))$ is empirical process, analogous to the proof of Theorem 2, using Theorem 8.3 (the uniform law of large numbers) of Pollard (1990), we can obtain

$$
\sup _{u \in T}\left\|n^{-1} \sum_{i=1}^{n} d M_{i}(u) / S_{n, 0}^{*}(u, \beta(u))\right\| \rightarrow 0, \quad \text { as } \quad n \rightarrow \infty .
$$

Under Conditions 1-5, $\widehat{\mu}_{0}(u, \widehat{\beta}(u))+\mu_{0}(u)$ is bounded, in conjunction with (B56), (B57), (B58) and (B59), we obtain

$$
\sup _{u \in T}\left\|\widehat{\mu}_{0}^{2}(u, \widehat{\beta}(u))-\mu_{0}^{2}(u)\right\| \rightarrow 0, \quad \text { as } \quad n \rightarrow \infty .
$$

Therefore, the second term converges to zero.

For the third term of the right-hand side, from (B51), obviously, converges to zero.

Hence,

$$
\left\|\widehat{\Sigma}_{2}(t)-\Sigma_{2}(t)\right\| \rightarrow 0, \quad \text { as } \quad n \rightarrow \infty .
$$

Therefore, from (B49) and (B61), we have $\widehat{\Sigma}(t)$ is consistent.

Proof of the asymptotic normality of $\widehat{\mu}_{0}(t, \widehat{\beta}(t))$. Let

$$
\begin{aligned}
(n h)^{1 / 2}\left(\widehat{\mu}_{0}(t, \widehat{\beta}(t))-\mu_{0}(t)\right) & =(n h)^{1 / 2}\left(\widehat{\mu}_{0}(t, \widehat{\beta}(t))-\widehat{\mu}_{0}(t, \beta(t))\right)+(n h)^{1 / 2}\left(\widehat{\mu}_{0}(t, \beta(t))-\mu_{0}(t)\right) \\
& =H_{n}\left(t, \beta^{*}\right)(n h)^{1 / 2}(\widehat{\beta}(t)-\beta(t))+(n h)^{1 / 2} \sum_{i=1}^{n} d M_{i}(t) / n S_{n, 0}^{*}(t, \beta(t)) .
\end{aligned}
$$

where $H_{n}\left(t, \beta^{*}\right)=-S_{n, 1}^{*}\left(t, \beta^{*}\right) \sum_{i=1}^{n} I\left(C_{i} \geq t\right) N_{i}(t) o_{i}(t) / n S_{n, 0}^{* 2}\left(t, \beta^{*}\right)$, and $\beta^{*}$ is between $\widehat{\beta}(t)$ and $\beta(t)$.

For the second term of right-hand side of (B62), let

$$
\begin{aligned}
& (n h)^{1 / 2} \sum_{i=1}^{n} d M_{i}(t) / n S_{n, 0}^{*}(t, \beta(t)) \\
& =(n h)^{1 / 2} \sum_{i=1}^{n} d M_{i}(t) / n S_{0}^{*}(t, \beta(t))+n^{-1}(n h)^{1 / 2} \sum_{i=1}^{n}\left\{S_{n, 0}^{*-1}(t, \beta(t))-S_{0}^{*-1}(t, \beta(t))\right\} d M_{i}(t) .
\end{aligned}
$$

Define

$$
U_{3}(s)=n^{-1}(n h)^{1 / 2} \sum_{i=1}^{n} d M_{i}(s)
$$


Clearly, $E\left[U_{3}(s)\right]=0$.

Analogous to the proof of Lemma B3, using the functional central limit theorem of Pollard (1990), we shall argument $U_{3}$ converges to Gaussian process $\xi_{3}$. Now we test the conditions (i)-(v) in Pollard (1990). Under Conditions $1-5$, by Lemma A1, we have, for any $s_{1}, s_{2} \in T$,

$$
\begin{aligned}
& \lim _{n \rightarrow \infty} E\left(U_{3}\left(s_{1}\right) U_{3}\left(s_{2}\right)\right) \\
& =\lim _{n \rightarrow \infty} E\left(n^{-1} h \sum_{i=1}^{n}\left(d M_{i}\left(s_{1}\right) d M_{i}\left(s_{2}\right)\right)\right) \\
& =h E\left(\left\{I\left(C \geq s_{1}\right) \mu_{0}\left(s_{1}\right) \exp \left(\beta\left(s_{1}\right) z\right) o\left(s_{1}\right)\right\}\left\{I\left(C \geq s_{2}\right) \mu_{0}\left(s_{2}\right) \exp \left(\beta\left(s_{2}\right) z\right) o\left(s_{2}\right)\right\}\right)+o_{p}(1) \\
& = \begin{cases}h E\left(p_{1}(s \mid z) p_{2}(s \mid z) \mu_{0}^{2}(s) \exp (2 \beta(s) z)\right)+o_{p}(1) & s_{1}=s_{2}=s \\
0 & s_{1} \neq s_{2}\end{cases}
\end{aligned}
$$

Then, by the classical multivariate central limit theorem for independent random vectors, the finitedimensional distributions of $U_{3}$ converge to those of gaussian process $\xi_{3}$, which converge to zero, as $h$ gets to zero. Thus condition (ii) holds. Next, checking the tightness. Under Conditions 1-5, we know $\left\{I\left(C_{i} \geq t\right) N_{i}(t) o_{i}(t), t \in T\right\}$ has finite points, and $\exp \left(\beta(t) z_{i}\right) o_{i}(t)$ are bounded variation functions. Thus, $\left\{d M_{i}(t), t \in T\right\}$ is manageable, and the envelops can be chosen as constant $\bar{B} / \sqrt{ } n$, then (i)(iii)(iv) holds.To verify (v), for any $s_{1}, s_{2} \in T$, define

$$
\rho_{n}\left(s_{1}, s_{2}\right)=E\left(U_{3}\left(s_{1}\right)-U_{3}\left(s_{2}\right)\right)^{2}, \quad \rho\left(s_{1}, s_{2}\right)=E\left(\xi_{3}\left(s_{1}\right)-\xi_{3}\left(s_{2}\right)\right)^{2} .
$$

Further,

$$
\begin{aligned}
& \rho_{n}\left(s_{1}, s_{2}\right)=E\left(n^{-1} \sqrt{n h} \sum_{i=1}^{n}\left(d M_{i}\left(s_{1}\right)-d M_{i}\left(s_{2}\right)\right)\right)^{2} \\
& =n^{-1} h \sum_{i=1}^{n} E\left(I\left(C_{i} \geq s_{1}\right) \mu_{0}^{2}\left(s_{1}\right) \exp \left(2 \beta\left(s_{1}\right) z_{i}\right) o_{i}\left(s_{1}\right)+I\left(C_{i} \geq s_{2}\right) \mu_{0}^{2}\left(s_{2}\right) \exp \left(2 \beta\left(s_{2}\right) z_{i}\right) o_{i}\left(s_{2}\right)\right) .
\end{aligned}
$$

Clearly, $\left\{\rho_{n}\right\}$ is equicontinuous on $T$, and $\lim _{n \rightarrow \infty} \rho_{n}\left(s_{1}, s_{2}\right)=\rho\left(s_{1}, s_{2}\right), \rho$ is pseudometric on $T$. Thus $\rho_{n}$ converges, uniformly on $T$, to $\rho$. And, let $\left\{s_{1}^{(n)}\right\},\left\{s_{2}^{(n)}\right\}$ be any two sequence in $T$, it follows that if $\rho\left(s_{1}^{(n)}, s_{2}^{(n)}\right) \rightarrow 0$, then $\rho_{n}\left(s_{1}^{(n)}, s_{2}^{(n)}\right) \rightarrow 0$, then (v) holds. Therefore, $U_{3}$ converges in distribution to Gaussian process on $T$, and covariance matrix is diagonal matrix, and the matrix is zero, when $h$ gets to zero. That is, $U_{3}$ converges in distribution zero, as $n \rightarrow \infty, h \rightarrow 0$ and $n h \rightarrow \infty$. Moreover, using the Strong Representation Theorem of Pollard (1990), we have a new probability space and

$$
\sup _{s \in T}\left\|U_{3}(s)-0\right\| \rightarrow 0, \quad \text { as } \quad n \rightarrow \infty .
$$

By Lemma A1 and Conditions 1-5, we can obtain

$$
\sup _{s \in T}\left\|S_{n, 0}^{*-1}(t, \beta(t))-S_{0}^{*-1}(t, \beta(t))\right\| \rightarrow 0, \quad \text { as } \quad n \rightarrow \infty .
$$

Then, by Lemma A2 combined with (B65) and (B66), we can derive, in probability,

$$
n^{-1}(n h)^{1 / 2} \sum_{i=1}^{n}\left\{S_{n, 0}^{*-1}(t, \beta(t))-S_{0}^{*-1}(t, \beta(t))\right\} d M_{i}(t) \rightarrow 0, \quad \text { as } \quad n \rightarrow \infty .
$$

which holds in the original probability space. And in analogy with the prove of $U_{3}(s)$, we can check that, in distribution,

$$
(n h)^{1 / 2} \sum_{i=1}^{n} d M_{i}(t) / n S_{0}^{*}(t, \beta(t)) \rightarrow 0, \quad \text { as } \quad n \rightarrow \infty .
$$


Therefore, from (B63), (B67) and (B68), we can obtain, in probability,

$$
(n h)^{1 / 2} \sum_{i=1}^{n} d M_{i}(t) / n S_{n, 0}^{*}(t, \beta(t)) \rightarrow 0, \quad \text { as } \quad n \rightarrow \infty .
$$

For the first term of right-hand side of (B62). Under Conditions 1-5 and Lemma A1, form (14), we obtain

$$
H_{n}\left(t, \beta^{*}\right) \rightarrow-q_{1}(t) / q_{0}(t), \quad n \rightarrow \infty .
$$

By the assumption of $n h^{5}=o(1)$, from (16), we can derive

$$
(n h)^{1 / 2}(\widehat{\beta}(t)-\beta(t)) \rightarrow N\left(0, \nu_{0} \sigma_{1}^{-2}(t) \sigma_{2}(t)\right), \quad \text { as } \quad n \rightarrow \infty .
$$

Therefore, from (B70) and (B71), we have

$$
H_{n}\left(t, \beta^{*}\right)(n h)^{1 / 2}(\widehat{\beta}(t)-\beta(t)) \rightarrow N\left(\nu_{0} q_{0}^{-2}(t) q_{1}(t) \sigma_{1}^{-2}(t) \sigma_{2}(t)\right), \quad \text { as } \quad n \rightarrow \infty .
$$

Hence, form (B62), (B69) and (B72), using Slutsky's theorem, we can obtain

$$
(n h)^{1 / 2}\left(\widehat{\mu}_{0}(t, \widehat{\beta}(t))-\mu_{0}(t)\right) \rightarrow N\left(0, \Sigma_{3}(t)\right), \quad n \rightarrow \infty,
$$

where $\Sigma_{3}(t)=\nu_{0} q_{0}^{-2}(t) q_{1}(t) \sigma_{1}^{-2}(t) \sigma_{2}(t)$.

\section{APPENDIX 3}

Here, we will show the simulation results about the local kernel estimators $\widehat{\beta}(t)$ with corresponding setting that $\beta(t)=\sqrt{ } t$ and $\beta(t)=0 \cdot 5(\operatorname{Beta}(x / 12,3,3)+\operatorname{Beta}(x / 12,4,4))$, respectively, under sample sizes equal to 500. Those figures are displayed in the end of this paper.

\section{REFERENCES}

BILIAS, Y., GU, M. \& YING, Z. (1997). Towards a general asymptotic theory for cox model with staggered entry. The Annals of Statistics 25, 662-682.

BRESLOW, N. (1974). Covariance analysis of censored survival data. Biometrics 30, 89.

Buchiolz, A. \& SAUERBReI, W. (2011). Comparison of procedures to assess nonlinear and timevarying effects in multivariable models for survival data. Biometrical Journal 53, 308-331.

CAI, J., FAN, J., ZHOU, H. \& ZHOU, Y. (2007). Hazard models with varying coefficients for multivariate failure time data. The Annals of Statistics 35, 324-354.

CAI, Z., FAN, J. \& LI, R. (2000). Efficient estimation and inferences for varying-coefficient models. Journal of the American Statistical Association 95, 888-902.

CAI, Z. \& SUN, Y. (2003). Local linear estimation for timedependent coefficients in cox's regression models. Scandinavian Journal of Statistics 30, 93-111.

Claeskens, G. \& Van Keilegom, I. (2003). Bootstrap confidence bands for regression curves and their derivatives. The Annals of Statistics 31, 1852-1884.

Cox, D. R. (1992). Regression Models and Life-Tables. Springer New York.

FAN, J. C. C. \& GiJBELS, R. (1996). Local polynomial modelling and its applications. Chapman and Hall.

Groux, H., Bigler, M., De VRies, J. E. \& RonCARolo, M.-G. (1998). Inhibitory and stimulatory effects of il-10 on human cd8+ t cells. The Journal of Immunology 160, 3188-3193.

Hardle, W., JANSSEn, P. \& Serfling, R. (1988). Strong uniform consistency rates for estimators of conditional functionals. The Annals of Statistics 16, 1428-1449.

He, X., Tong, X., Sun, J. \& Cook, R. J. (2007). Regression analysis of multivariate panel count data. Biostatistics 9, 234-48.

Hoover, D. R., Rice, J. A., Wu, C. O. \& YAnG, L.-P. (1998). Nonparametric smoothing estimates of timevarying coefficient models with longitudinal data. Biometrika 85, 809-822.

HU, X. J., SUn, J. \& WEI, L. (2003). Regression parameter estimation from panel counts. Scandinavian Journal of Statistics 30, 25-43.

KeARley, J., BARKer, J. E., Robinson, D. S. \& Lloyd, C. M. (2005). Resolution of airway inflammation and hyperreactivity after in vivo transfer of $\mathrm{cd} 4+\mathrm{cd} 25+$ regulatory t cells is interleukin 10 dependent. Journal of Experimental Medicine 202, 1539-1547. 
Lawless, J. F. \& NAdeaU, C. (1995). Some simple robust methods for the analysis of recurrent events. Technometrics 37, 158-168.

LEI, H., YING, Z. \& WANZHU, T. (2014). A spline-based semiparametric sieve likelihood method for over-dispersed panel count data. Canadian Journal of Statistics 42, 217-245.

LI, N., PARK, D.-H., SUN, J. \& KIM, K. (2011). Semiparametric transformation models for multivariate panel count data with dependent observation process. Canadian Journal of Statistics 39, 458-474.

LI, N., SUN, L. \& SUN, J. (2010). Semiparametric transformation models for panel count data with dependent observation process. Statistics in Biosciences 2, 191-210.

Li, Y., He, X., WANG, H., Zhang, B. \& Sun, J. (2015). Semiparametric regression of multivariate panel count data with informative observation times. Journal of Multivariate Analysis 140, 209-219.

LIN, H., FEI, Z. \& LI, Y. (2016). A semiparametrically efficient estimator of the timevarying effects for survival data with timedependent treatment. Scandinavian Journal of Statistics 43, 649-663.

Perperoglou, A. (2013). Reduced rank hazard regression with fixed and timevarying effects of the covariates. Biometrical Journal 55, 38-51.

Perperoglou, A. (2014). Cox models with dynamic ridge penalties on timevarying effects of the covariates. Statistics in medicine 33, 170-180.

Pollard, D. (1990). Empirical processes: Theory and Applications, vol. 2. Paul Shaman, Jessica Utts and Jose L. Gonzalez.

Sun, J., Tong, X. \& He, X. (2007). Regression analysis of panel count data with dependent observation times. Biometrics 63, 1053-1059.

Tepper, R. S., Llapur, C. J., Jones, M. H., Tiller, C., Coates, C., Kimmel, R., Kisling, J., Katz, B., DING, Y. \& SWIGONSKI, N. (2008). Expired nitric oxide and airway reactivity in infants at risk for asthma. Journal of Allergy and Clinical Immunology 122, 760-765.

Tian, L., ZuCKer, D. \& WeI, L. J. (2005). On the cox model with time-varying regression coefficients. Publications of the American Statistical Association 100, 172-183.

Wellner, J. A. \& ZhANG, Y. (2007). Two likelihood-based semiparametric estimation methods for panel count data with covariates. Ann. Statist. 35, 2106-2142.

Yao, W., Barbé-Tuana, F. M., Llapur, C. J., Jones, M. H., Tiller, C., Kimmel, R., Kisling, J., NguYen, E. T., NGuYEn, J. \& YU, Z. (2010). Evaluation of airway reactivity and immune characteristics as risk factors for wheezing early in life. Journal of Allergy and Clinical Immunology 126, 483-488. e1.

YU, Z. \& LIN, X. (2010). Semiparametric regression with time-dependent coefficients for failure time data analysis. Stat Sin 20, 853-869.

ZHANG, Y. (2002). A semiparametric pseudolikelihood estimation method for panel count data. Biometrika 89, $39-48$.

ZhaO, H., TU, W. \& YU, Z. (2018). A nonparametric time-varying coefficient model for panel count data. Journal of Nonparametric Statistics , 1-22.

ZHAO, P.-L. (1994). Asymptotics of kernel estimators based on local maximum likelihood. Journaltitle of Nonparametric Statistics 4, 79-90.

Zhu, L., Zhang, Y., Li, Y., Sun, J. \& Robison, L. L. (2018). A semiparametric likelihoodbased method for regression analysis of mixed panelcount data. Biometrics $\mathbf{7 4}, 488-497$. 
(c1)

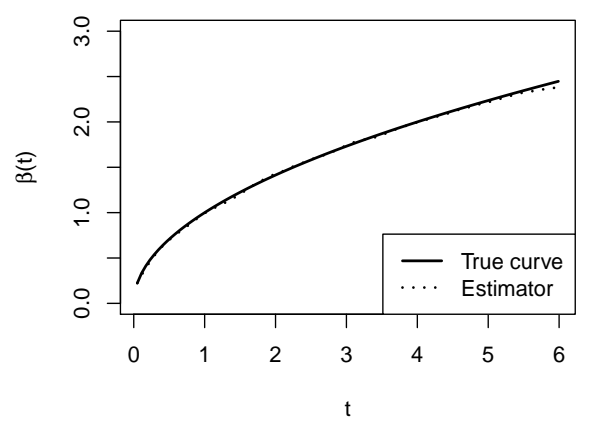

(c3)

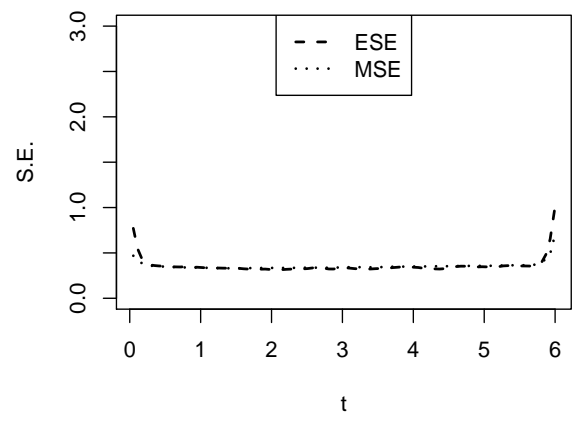

(c5)

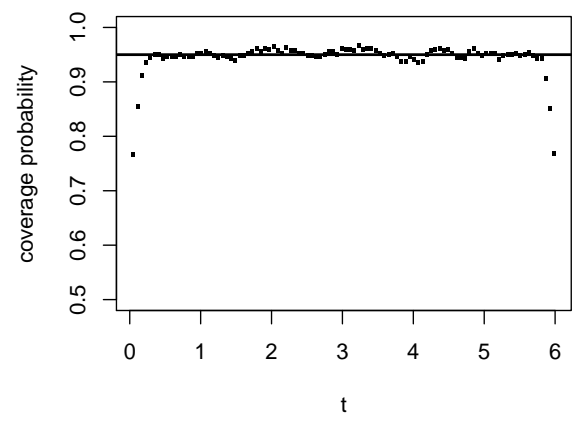

(c2)

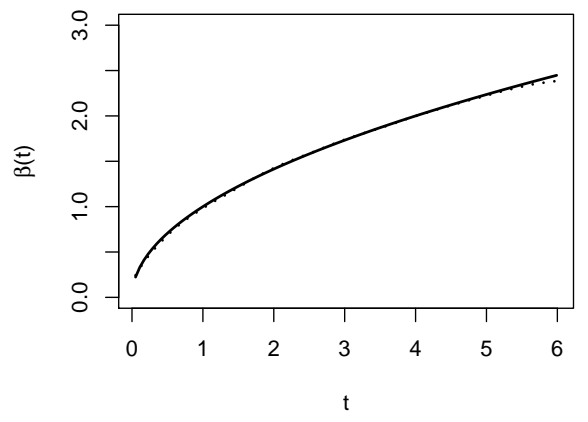

(c4)

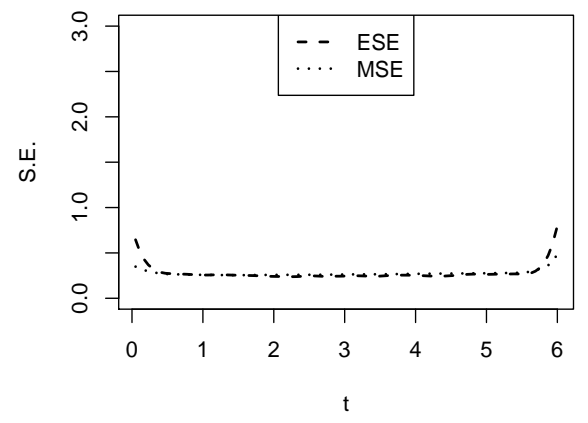

(c6)

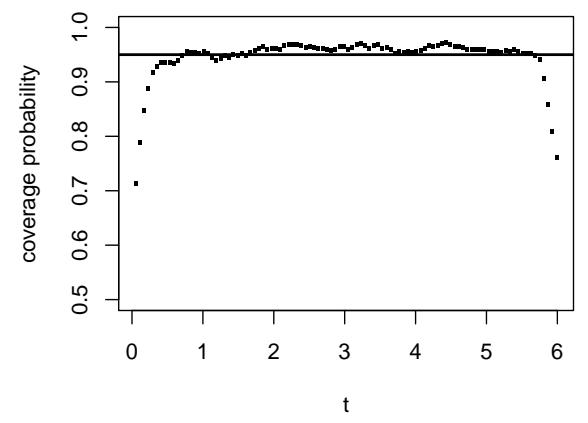

Fig. 4: (c1) and (c2): The true and the average of the local kernel estimator with bandwidth $0 \cdot 3$ and $0 \cdot 5$, respectively. (c3) and (c4): Comparison of empirical standard errors (ESE) and the estimated standard errors (MSE) for $\widehat{\beta}(t)$ with bandwidth 0.3 and 0.5 , respectively; (c5) and (c6): Empirical coverage probabilities of the $95 \%$ confidence intervals for $\widehat{\beta}(t)$ with bandwidth $0 \cdot 3$ and $0 \cdot 5$, respectively. 
(d1)

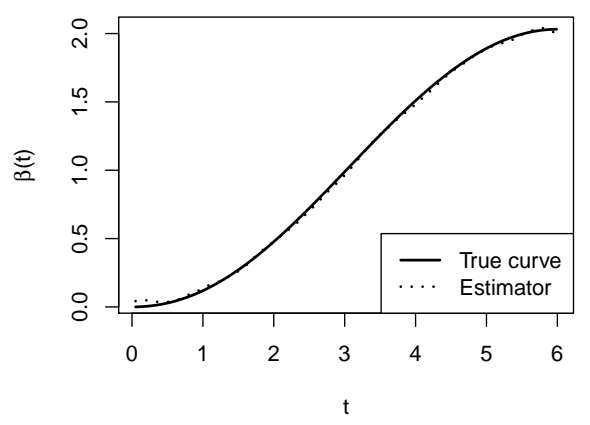

(d3)

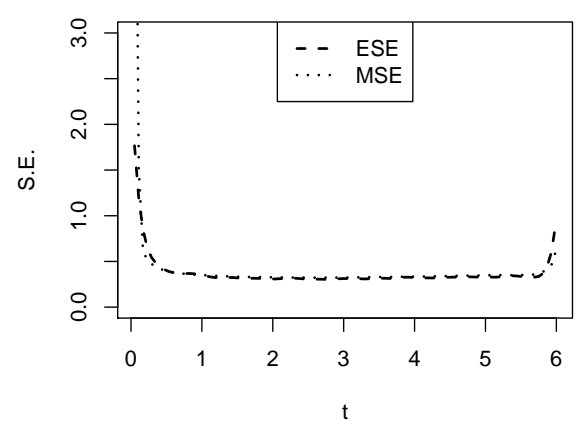

(d5)

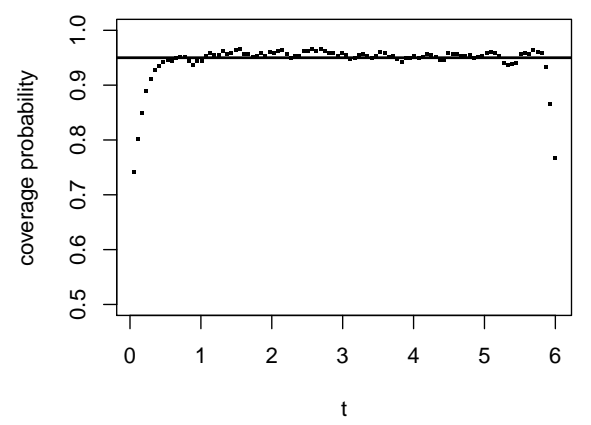

(d2)

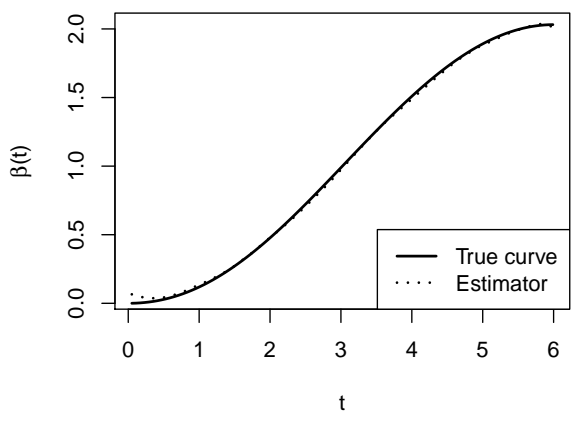

(d4)

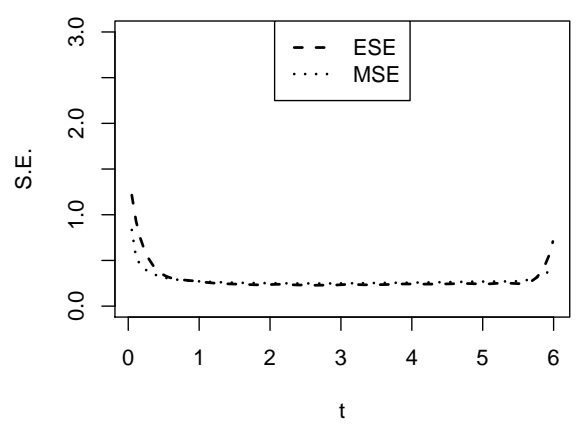

(d6)

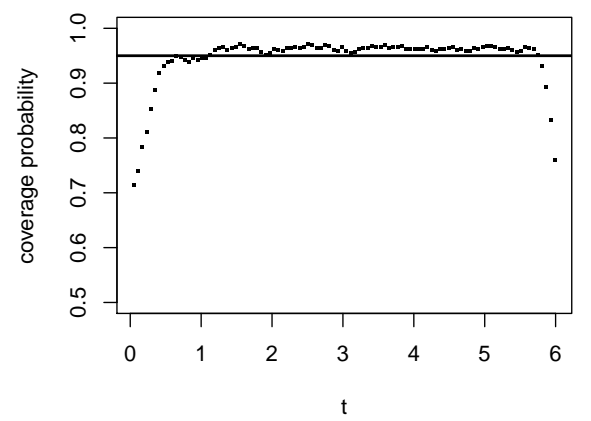

Fig. 5: (d1) and (d2): The true and the average of the local kernel estimator with bandwidth $0 \cdot 3$ and $0 \cdot 5$, respectively. (c3) and (c4): Comparison of empirical standard errors (ESE) and the estimated standard errors (MSE) of $\widehat{\beta}(t)$ with bandwidth $0 \cdot 3$ and 0.5 , respectively; (d5) and (d6): Empirical coverage probabilities of the $95 \%$ confidence intervals for $\widehat{\beta}(t)$ with bandwidth $0 \cdot 3$ and $0 \cdot 5$, respectively. 